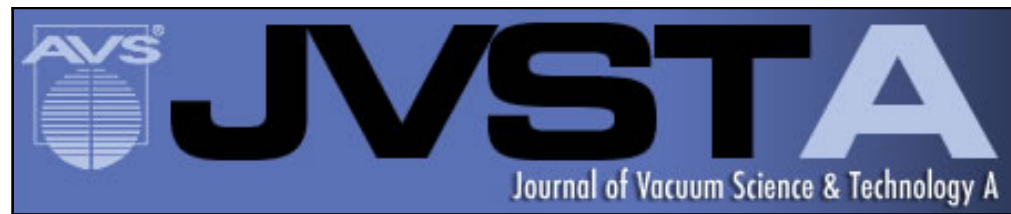

\title{
Modeling and experimental investigation of the plasma uniformity in CF4/O2 capacitively coupled plasmas, operating in single frequency and dual frequency regime
}

Yu-Ru Zhang, Stefan Tinck, Peter De Schepper, You-Nian Wang, and Annemie Bogaerts

Citation: Journal of Vacuum Science \& Technology A 33, 021310 (2015); doi: 10.1116/1.4906819

View online: http://dx.doi.org/10.1116/1.4906819

View Table of Contents: http://scitation.aip.org/content/avs/journal/jvsta/33/2?ver=pdfcov

Published by the AVS: Science \& Technology of Materials, Interfaces, and Processing

\section{Articles you may be interested in}

Experimental investigations of electron density and ion energy distributions in dual-frequency capacitively coupled plasmas for Ar/CF 4 and $\mathrm{Ar} / \mathrm{O} 2 / \mathrm{CF} 4$ discharges

J. Appl. Phys. 115, 013301 (2014); 10.1063/1.4859595

Experimental and numerical investigations of electron density in low-pressure dual-frequency capacitively coupled oxygen discharges

J. Vac. Sci. Technol. A 31, 061308 (2013); 10.1116/1.4822059

Numerical study of ion energy and angular distributions in dualfrequency capacitively coupled CF4 plasmas Phys. Plasmas 19, 023506 (2012); 10.1063/1.3684675

Numerical investigation of ion energy distribution and ion angle distribution in a dual-frequency capacitively coupled plasma with a hybrid model

Phys. Plasmas 14, 113501 (2007); 10.1063/1.2780136

Numerical study of $\mathrm{Ar}$ / CF 4 / N 2 discharges in single- and dual-frequency capacitively coupled plasma reactors J. Appl. Phys. 94, 3748 (2003); 10.1063/1.1603348

\section{Instruments for Advanced Science} w www.HidenAnalytical.com E info@hiden.co.uk CLICK TO VIEW our product catalogue

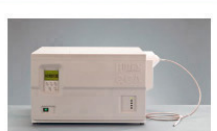

Gas Analysis

dynamic measurement of reaction gas streans catalysis and thermal analysis molecular beam studies dissolved species probes fermentation environmental and ecological stud

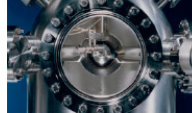

Surface Science

UHVTPD

SIMS elemental imaging - surface mapping

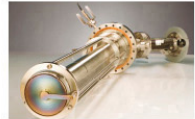

Plasma Diagnostics plasma source characterization etch and deposition process reaction analysis of neutral and radical species

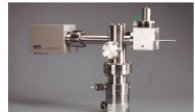

Vacuum Analysis partial pressure measurement and control of process gases reactive sputter process contro vacuum diagnostics Teum coatin proses monitorin 


\title{
Modeling and experimental investigation of the plasma uniformity in $\mathrm{CF}_{4} / \mathrm{O}_{2}$ capacitively coupled plasmas, operating in single frequency and dual frequency regime
}

\author{
Yu-Ru Zhang a) \\ Key Laboratory of Materials Modification by Laser, Ion, and Electron Beams (Ministry of Education), \\ School of Physics and Optoelectronic Technology, Dalian University of Technology, Dalian 116024, \\ People's Republic of China and Department of Chemistry, University of Antwerp, Campus Drie Eiken, \\ Universiteitsplein 1, BE-2610 Wilrijk-Antwerp, Belgium \\ Stefan Tinck \\ Department of Chemistry, University of Antwerp, Campus Drie Eiken, Universiteitsplein 1, \\ BE-2610 Wilrijk-Antwerp, Belgium \\ Peter De Schepper \\ Department of Chemistry, University of Leuven, Kapeldreef, BE-3001 Leuven, Belgium and IMEC, \\ Kapeldreef 75, BE-3001 Leuven, Belgium \\ You-Nian Wang \\ Key Laboratory of Materials Modification by Laser, Ion, and Electron Beams (Ministry of Education), \\ School of Physics and Optoelectronic Technology, Dalian University of Technology, Dalian 116024, \\ People's Republic of China \\ Annemie Bogaerts \\ Department of Chemistry, University of Antwerp, Campus Drie Eiken, Universiteitsplein 1, \\ BE-2610 Wilrijk-Antwerp, Belgium
}

(Received 17 August 2014; accepted 16 January 2015; published 29 January 2015)

\begin{abstract}
A two-dimensional hybrid Monte Carlo-fluid model, incorporating a full-wave solution of Maxwell's equations, is employed to describe the behavior of high frequency (HF) and very high frequency capacitively coupled plasmas (CCPs), operating both at single frequency (SF) and dual frequency (DF) in a $\mathrm{CF}_{4} / \mathrm{O}_{2}$ gas mixture. First, the authors investigate the plasma composition, and the simulations reveal that besides $\mathrm{CF}_{4}$ and $\mathrm{O}_{2}$, also $\mathrm{COF}_{2}, \mathrm{CF}_{3}$, and $\mathrm{CO}_{2}$ are important neutral species, and $\mathrm{CF}_{3}^{+}$ and $\mathrm{F}^{-}$are the most important positive and negative ions. Second, by comparing the results of the model with and without taking into account the electromagnetic effects for a SF CCP, it is clear that the electromagnetic effects are important, both at 27 and $60 \mathrm{MHz}$, because they affect the absolute values of the calculation results and also (to some extent) the spatial profiles, which accordingly affects the uniformity in plasma processing. In order to improve the plasma radial uniformity, which is important for the etch process, a low frequency (LF) source is added to the discharge. Therefore, in the major part of the paper, the plasma uniformity is investigated for both SF and DF CCPs, operating at a HF of 27 and $60 \mathrm{MHz}$ and a LF of $2 \mathrm{MHz}$. For this purpose, the authors measure the etch rates as a function of position on the wafer in a wide range of LF powers, and the authors compare them with the calculated fluxes toward the wafer of the plasma species playing a role in the etch process, to explain the trends in the measured etch rate profiles. It is found that at a $\mathrm{HF}$ of $60 \mathrm{MHz}$, the uniformity of the etch rate is effectively improved by adding a LF power of $2 \mathrm{MHz}$ and $300 \mathrm{~W}$, while its absolute value increases by about $50 \%$, thus a high etch rate with a uniform distribution is observed under this condition. (C) 2015 American Vacuum Society. [http://dx.doi.org/10.1116/1.4906819]
\end{abstract}

\section{INTRODUCTION}

Plasma etching plays a vitally important role in modern integrated circuit fabrication technology. Carbon tetrafluoride $\left(\mathrm{CF}_{4}\right)$ is an industrially important gas, which is used in the selective and anisotropic removal of patterned silicon. ${ }^{1}$ For this purpose, the physical and chemical processes in $\mathrm{CF}_{4}$ plasmas need to be well understood to improve the plasma performance, and they have been widely investigated both experimentally ${ }^{2-6}$ and theoretically ${ }^{7-13}$ in capacitively coupled plasmas (CCPs). Besides measurements of the densities of

a)Electronic mail: yrzhang@dlut.edu.cn negative ions ${ }^{2}$ and high mass species, ${ }^{3}$ special attention has been paid to the production and loss mechanisms of $\mathrm{CF}_{\mathrm{x}}$ radicals by means of laser induced fluorescence ${ }^{4}$ and infrared absorption spectroscopy ${ }^{5,6}$ under various conditions, since these radials play an important role in etch and polymerization processes. Moreover, considerable effort has also been made to study the important plasma physics and chemistry in $\mathrm{CF}_{4}$ discharges (i.e., the role of negative ion attachment, the discharge mode transition, etc.) by fluid simulations ${ }^{7-10}$ and particle-in-cell/Monte Carlo modeling. ${ }^{11-13}$

In order to control the production of radicals and improve the selectivity in etching, $\mathrm{O}_{2}$ or other dilution gases are usually added to $\mathrm{CF}_{4}$ discharges for practical material 
processing. Mogab et al. ${ }^{14}$ showed that with an increasing $\mathrm{O}_{2}$ fraction in $\mathrm{CF}_{4}$ plasmas, the density of $\mathrm{F}$ atoms increases first because of the reaction between $\mathrm{O}_{2}$ and $\mathrm{CF}_{3}$ radicals, and subsequently, it decreases due to the oxygen dilution effects. Since the fraction of $\mathrm{O}_{2}$ in the mixture affects the $\mathrm{F}$ atom density, the etch rate is also influenced, and this is another reason why $\mathrm{O}_{2}$ is typically added as input gas. Besides experimental research, ${ }^{14-17}$ significant progress has also been achieved in theoretical studies of $\mathrm{CF}_{4} / \mathrm{O}_{2}$ plasmas. ${ }^{18-21}$ In most of these studies, the emphasis is put on the densities of neutral species and the etch rate as a function of $\mathrm{O}_{2}$ fraction, and the calculation results showed typically good agreement with experimental measurements.

In most of the studies mentioned above, the discharge is sustained by a single frequency (SF) source. However, in such SF discharges, the ion flux to the substrate and the energy of the ions when bombarding the substrate cannot be controlled independently in CCPs. Therefore, the dual frequency (DF) excitation system has been proposed to overcome this limitation and to meet the line-width, selectivity, and damage control demands for next-generation fabrication. ${ }^{1,22}$ Booth et al. ${ }^{23}$ observed experimentally a significant decrease of the $\mathrm{F}^{-}$density by means of cavity ring-down spectroscopy, when a low frequency (LF) power $(2 \mathrm{MHz})$ was added to the $27 \mathrm{MHz}$ power in $\mathrm{Ar} / \mathrm{CF}_{4}$ and $\mathrm{Ar} / \mathrm{CF}_{4} / \mathrm{O}_{2}$ plasmas. Furthermore, the electrical asymmetry effect ${ }^{24}$ and the electron energy distribution function $(\mathrm{EEDF})^{25}$ have been investigated numerically by a one-dimensional PIC simulation and a two-dimensional hydrodynamics model in $\mathrm{DF} \mathrm{CF} 4$ and $\mathrm{Ar} / \mathrm{CF}_{4} / \mathrm{O}_{2}$ discharges.

In order to obtain high density plasmas with low energy ions, very high frequency (VHF) sources have recently attracted growing interest and are now widely used in DF systems. However, in VHF discharges, when the excitation wavelength becomes comparable to the electrode dimension, the standing-wave effect becomes dominant, and it yields a pronounced power deposition at the reactor center. On the other hand, when the skin depth is not large compared with the plasma thickness, the skin effect has a significant influence, and results in a substantial power deposition at the radial edge. Therefore, both these so-called electromagnetic effects $^{26,27}$ significantly influence the plasma uniformity and therefore the etch uniformity in VHF discharges.

Recently, several experimental ${ }^{28-30}$ and theoretical ${ }^{30-32}$ studies have been published on the plasma characteristics in DF CCPs sustained by VHF sources. Hebner et $_{\text {al. }}{ }^{28}$ performed measurements in a DF $300 \mathrm{~mm}$-wafer plasma processing reactor, showing that the electron density was independent of the $13 \mathrm{MHz}$ bias power as it increased from 0 to $1500 \mathrm{~W}$ when the high frequency (HF) was fixed at $60 \mathrm{MHz}$. Barnat et al. ${ }^{29}$ revealed that the radial distribution of the voltage drop across the sheath became nonuniform at the frequency of $60 \mathrm{MHz}$, and the nonuniformity was still present when a LF (13.56 $\mathrm{MHz}$ ) source was applied to the electrode together with a HF $(60 \mathrm{MHz})$ source. Bera et al..$^{30}$ proved that, by a combination of computational modeling and diagnostic experiments, VHF mixing was an effective method for dynamically controlling the plasma uniformity. Furthermore, Yang and Kushner ${ }^{31,32}$ utilized a full-wave Maxwell solver, focusing on the plasma characteristics in DF CCPs at various discharge conditions. They reported that the electron density shifts from edge high to center-and-edge high with increasing HF, and finally, a midradius high profile was observed at $150 \mathrm{MHz}$ in $\mathrm{Ar} / \mathrm{CF}_{4} \mathrm{CCPs}$.

From the literature overview mentioned above, it is indeed clear that when the discharge is sustained by VHF sources, the so-called electromagnetic effects start to have a significant influence on the plasma radial uniformity, which accordingly affects the etch and deposition processes. To improve the spatial uniformity, many methods have been put forward, for instance, lens-shaped electrodes, ${ }^{33-35}$ ladder-shaped electrodes, ${ }^{36,37}$ graded conductivity electrodes, ${ }^{38}$ and phase-shift control. ${ }^{39-42}$ Recently, Chabert and Braithwaite ${ }^{43}$ proposed that adding a LF source could suppress the nonuniformity caused by the VHF source. Therefore, the aim of this work is to elucidate the influence of the LF power on the plasma radial uniformity, which has not been investigated in detail yet in a $\mathrm{CF}_{4} / \mathrm{O}_{2}$ gas mixture. For this purpose, we employ a two-dimensional hybrid fluid-Monte Carlo model, i.e., the so-called hybrid plasma equipment model (HPEM) ${ }^{44}$ to investigate the plasma characteristics in $\mathrm{CF}_{4} / \mathrm{O}_{2} \mathrm{DF}$ discharges. The etch rate and its uniformity across the wafer are also measured experimentally, and the model is used to explain the trends in the etch rate uniformity. Indeed, a better understanding of the $\mathrm{CF}_{4} / \mathrm{O}_{2} \mathrm{HF}$ and $\mathrm{VHF}$ plasma is important, and can help us to effectively improve the plasma processing in microelectronics applications.

\section{DESCRIPTION OF THE MODEL}

In this paper, the so-called HPEM, developed by Kushner and co-workers ${ }^{44}$ is employed to investigate the electromagnetic effects in $\mathrm{DF} \mathrm{CF}_{4} / \mathrm{O}_{2} \mathrm{CCPs}$ under different discharge conditions. The HPEM is a comprehensive modeling platform, which addresses different physical and chemical processes in a self-consistent manner by a series of modules.

In this work, three modules of the HPEM, i.e., the fluid kinetics simulation (FKS) module, the electron energy transport module (EETM), as well as the Maxwell solver, are employed to investigate the plasma properties in a $\mathrm{CF}_{4} / \mathrm{O}_{2}$ gas mixture. The diagram of the different modules of the HPEM used in this work is shown in Fig. 1.

The EETM module computes the time varying electron transport properties, by using the electron Monte Carlo

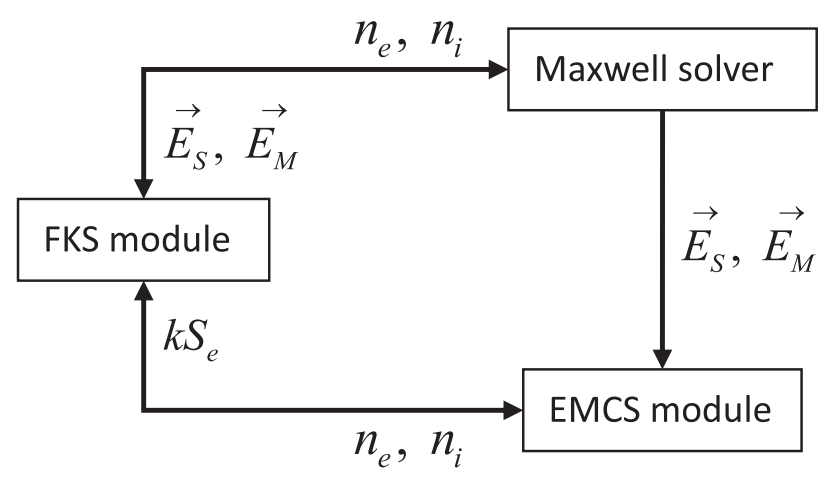

FIG. 1. Diagram of the different modules of the HPEM used in this work. 
simulation (EMCS) module or the Boltzmann equation. In this work, the EMCS module is used to simulate the electron temperature, EEDF and electron impact rate coefficients. The collisions between electrons and other species are included in the EMCS module, and the electron trajectories and positions are advanced according to Newton's law and by the electrostatic and electromagnetic fields calculated from the Maxwell solver.

The electron impact rate coefficients from the EMCS are used as input in the FKS module, in which the continuum transport equations are solved to obtain the plasma species densities and fluxes

$$
\frac{\partial n_{i}}{\partial t}+\nabla \cdot \boldsymbol{\Gamma}_{\mathbf{i}}=S_{i}
$$

where $n_{i}, \Gamma_{\mathbf{i}}$, and $S_{i}$ are the density, flux, and source term of particle $\mathrm{i}$.

The momentum and energy equations solved for neutral and ion transport are

$$
\begin{aligned}
\frac{\partial \Gamma_{\mathbf{i}}}{\partial t}= & \frac{\partial\left(n_{i} \mathbf{u}_{\mathbf{i}}\right)}{\partial t} \\
= & -\frac{1}{m_{i}} \nabla \cdot\left(n_{i} \mathbf{u}_{\mathbf{i}} \mathbf{u}_{\mathbf{i}}\right)+\frac{q_{i} n_{i}}{m_{i}} \mathbf{E}-\nabla \cdot \overline{\overline{\mu_{i}}} \\
& -\sum_{j} \frac{m_{j}}{m_{i}+m_{j}} n_{i} n_{j}\left(\mathbf{u}_{\mathbf{i}}-\mathbf{u}_{\mathbf{j}}\right) \nu_{i j}, \\
\frac{\partial\left(n_{i} c_{i} T_{i}\right)}{\partial t}= & \nabla \cdot \overline{\bar{\kappa}} \nabla T_{i}-P_{i} \nabla \cdot \mathbf{u}_{\mathbf{i}}-\nabla \cdot\left(n_{i} \mathbf{u}_{\mathbf{i}} \varepsilon_{i}\right)+\frac{n_{i} q_{i}{ }^{2}}{m_{i} u_{i}} E^{2} \\
& \quad+\sum_{j} 3 \frac{m_{i j}}{m_{i}+m_{j}} n_{i} n_{j} \nu_{i j} k_{B}\left(T_{j}-T_{i}\right) \\
& \quad \pm \sum_{j} 3 n_{i} n_{j} k_{i j} k_{B} T_{j} .
\end{aligned}
$$

Here, $\mathbf{u}_{\mathbf{i}}, m_{i}, T_{i}, q_{i}, \overline{\overline{\mu_{i}}}, c_{i}, P_{i}$, and $\varepsilon_{i}$ are the velocity, mass, temperature, charge, viscosity (used only for neutral species), heat capacity, pressure, and energy of particle $i$, respectively; $\nu_{i j}$ is the collision frequency between species $i$ and $j$, and $k_{i j}$ is the rate coefficient for formation of the species by collisions; $\overline{\bar{\kappa}}$ is the thermal conductivity.

In order to include the electromagnetic effects in VHF discharges, a full solution of Maxwell's equations is integrated into the FKS, as opposed to only the electrostatic Poisson's equation

$$
\begin{aligned}
& \frac{\partial E_{r}}{\partial z}-\frac{\partial E_{z}}{\partial r}=-\frac{\partial B_{\theta}}{\partial t}, \\
& -\frac{\partial B_{\theta}}{\partial z}=\mu J_{r}+\varepsilon \mu \frac{\partial E_{r}}{\partial t}, \\
& -\frac{1}{r} \frac{\partial\left(r B_{\theta}\right)}{\partial r}=\mu J_{z}+\varepsilon \mu \frac{\partial E_{z}}{\partial t},
\end{aligned}
$$

where $J_{r}$ and $J_{z}$ are the radial and axial components of the conduction current, $\mu$ is the permeability, and $\varepsilon$ is the permittivity.

It should be noted that the Darwin approach would also be sufficient for studying nearly all cases of the electromagnetic effects in high frequency CCP discharges, since the radiative
TABLE I. Overview of the species included in the model.

\begin{tabular}{lccc}
\hline \hline $\begin{array}{l}\text { Ground state } \\
\text { neutral species }\end{array}$ & $\begin{array}{c}\text { Excited } \\
\text { species }\end{array}$ & Positive ions & $\begin{array}{c}\text { Negatively } \\
\text { charged } \\
\text { species }\end{array}$ \\
\hline $\mathrm{CF}_{4}, \mathrm{CF}_{3}, \mathrm{CF}_{2}, \mathrm{CF}, \mathrm{C}, \mathrm{F}, \mathrm{F}_{2}$ & $\mathrm{~F}^{*}$ & $\mathrm{CF}_{3}^{+}, \mathrm{CF}_{2}^{+}, \mathrm{CF}^{+}, \mathrm{F}^{+}, \mathrm{F}_{2}^{+}$ & $\mathrm{CF}_{3}^{-}, \mathrm{F}^{-}$ \\
$\mathrm{O}_{2}, \mathrm{O}$, & $\mathrm{O}^{*}$ & $\mathrm{O}_{2}^{+}, \mathrm{O}^{+}$ & $\begin{array}{c}\mathrm{O}^{-} \\
\mathrm{CO}_{2}, \mathrm{CO}, \mathrm{COF}, \mathrm{COF}_{2}, \mathrm{FO}\end{array}$ \\
\hline \hline
\end{tabular}

effects can be neglected under this condition. ${ }^{58}$ By using the Darwin approximation, the courant criterion is removed, which could significantly reduce the computational cost. However, we adopted the full Maxwell solver in this paper, which may be not very necessary, but it is certainly valid. With this method, we can correctly include all the electromagnetic effects.

Subsequently, the inductive electric and electrostatic fields from the Maxwell solver are returned to the EMCS module to update the coordinates and the velocities of the electrons. The Maxwell equations are discretized on a staggered mesh and solved using the finite difference time domain techniques. In order to overcome the time limitation, Poisson's equation is solved by using the semi-implicit technique. Some numerical acceleration techniques have been employed to achieve the steady state. For instance, we could extrapolate the densities into the future by using the past history of densities. The iteration between different modules continues until convergence is achieved.

The plasma species included in the model are listed in Table I. In $\mathrm{CF}_{4} / \mathrm{O}_{2}$ plasmas, various molecules, radicals, ions, excited species, as well as the electrons exist, and need to be taken into account. The excited species $\mathrm{O}^{*}$ consists of the $4 \mathrm{~s}$ and $4 \mathrm{p}$ levels. Electron impact vibrational excitations are included for $\mathrm{O}_{2}$, as they contribute to the energy loss of the electrons, but the excited levels of $\mathrm{O}_{2}$ are not explicitly taken into account as separate species. Therefore, $\mathrm{O}_{2}$ includes the ground state molecule and two electronic excited levels with thresholds of 8.40 and $10.0 \mathrm{eV}$; for these three states, one rotational excitation and two vibrational excitations are included. ${ }^{45-47}$

The electron impact reactions considered in the model for the $\mathrm{CF}_{4} / \mathrm{O}_{2}$ plasma are presented in Table II. This table includes the electron collisions with various molecules, radicals, excited species, and ions, i.e., excitation, ionization, recombination, detachment, de-excitation, dissociation, as well as dissociative attachment, excitation, ionization, and recombination. Elastic collisions between the electrons and all the species are included in the model but are not listed in Table II. For most of the electron impact reactions, the rate coefficients are calculated from the energy dependent cross sections in the EMCS module, and the corresponding references for these cross sections are also given in Table II. The heavy particle reactions taken into account in the model are listed in Table III, as well as the rate coefficients, and the references where these data are adopted from.

A simple surface reaction mechanism is considered in this model. All positive ions are neutralized with a 
TABLE II. Electron impact reactions included in the model. (a) indicates the rate coefficient is calculated from the corresponding collision cross section.

\begin{tabular}{|c|c|c|}
\hline Reaction & Rate coefficient $\left(\mathrm{cm}^{3} / \mathrm{s}\right)$ & Reference \\
\hline $\mathrm{e}+\mathrm{CF}_{4} \rightarrow \mathrm{F}^{-}+\mathrm{CF}_{3}$ & (a) & 49 \\
\hline $\mathrm{e}+\mathrm{CF}_{4} \rightarrow \mathrm{CF}_{3}^{-}+\mathrm{F}$ & (a) & 49 \\
\hline $\mathrm{e}+\mathrm{CF}_{4} \rightarrow \mathrm{CF}_{3}+\mathrm{F}+\mathrm{e}$ & (a) & 49 \\
\hline $\mathrm{e}+\mathrm{CF}_{4} \rightarrow \mathrm{CF}_{2}+2 \mathrm{~F}+\mathrm{e}$ & (a) & 49 \\
\hline $\mathrm{e}+\mathrm{CF}_{4} \rightarrow \mathrm{CF}+\mathrm{F}+\mathrm{F}_{2}+\mathrm{e}$ & (a) & 49 \\
\hline $\mathrm{e}+\mathrm{CF}_{4} \rightarrow \mathrm{CF}_{3}^{+}+\mathrm{F}^{-}+\mathrm{e}$ & (a) & 49 \\
\hline $\mathrm{e}+\mathrm{CF}_{4} \rightarrow \mathrm{CF}_{3}^{+}+\mathrm{F}+2 \mathrm{e}$ & (a) & 49 \\
\hline $\mathrm{e}+\mathrm{CF}_{4} \rightarrow \mathrm{CF}_{3}^{+}+\mathrm{F}^{+}+3 \mathrm{e}$ & (a) & 49 \\
\hline $\mathrm{e}+\mathrm{CF}_{4} \rightarrow \mathrm{CF}_{2}^{+}+2 \mathrm{~F}+2 \mathrm{e}$ & (a) & 49 \\
\hline $\mathrm{e}+\mathrm{CF}_{4} \rightarrow \mathrm{CF}^{+}+\mathrm{F}+\mathrm{F}_{2}+2 \mathrm{e}$ & (a) & 49 \\
\hline $\mathrm{e}+\mathrm{CF}_{4} \rightarrow \mathrm{F}^{+}+\mathrm{CF}_{3}+2 \mathrm{e}$ & (a) & 49 \\
\hline $\mathrm{e}+\mathrm{CF}_{3} \rightarrow \mathrm{F}^{-}+\mathrm{CF}_{2}$ & (a) & 49 \\
\hline $\mathrm{e}+\mathrm{CF}_{3} \rightarrow \mathrm{CF}_{2}+\mathrm{F}+\mathrm{e}$ & (a) & 49 \\
\hline $\mathrm{e}+\mathrm{CF}_{3} \rightarrow \mathrm{CF}_{3}^{+}+2 \mathrm{e}$ & (a) & 49 \\
\hline $\mathrm{e}+\mathrm{CF}_{3} \rightarrow \mathrm{CF}_{2}^{+}+\mathrm{F}+2 \mathrm{e}$ & (a) & 49 \\
\hline $\mathrm{e}+\mathrm{CF}_{3} \rightarrow \mathrm{CF}^{+}+2 \mathrm{~F}+2 \mathrm{e}$ & (a) & 49 \\
\hline $\mathrm{e}+\mathrm{CF}_{3} \rightarrow \mathrm{F}^{+}+\mathrm{CF}_{2}+2 \mathrm{e}$ & (a) & 49 \\
\hline $\mathrm{e}+\mathrm{CF}_{2} \rightarrow \mathrm{F}^{-}+\mathrm{CF}$ & (a) & 49 \\
\hline $\mathrm{e}+\mathrm{CF}_{2} \rightarrow \mathrm{CF}+\mathrm{F}+\mathrm{e}$ & (a) & 49 \\
\hline $\mathrm{e}+\mathrm{CF}_{2} \rightarrow \mathrm{CF}_{2}^{+}+2 \mathrm{e}$ & (a) & 49 \\
\hline $\mathrm{e}+\mathrm{CF}_{2} \rightarrow \mathrm{CF}^{+}+\mathrm{F}+2 \mathrm{e}$ & (a) & 49 \\
\hline $\mathrm{e}+\mathrm{CF}_{2} \rightarrow \mathrm{F}^{+}+\mathrm{CF}+2 \mathrm{e}$ & (a) & 49 \\
\hline $\mathrm{e}+\mathrm{CF} \rightarrow \mathrm{C}+\mathrm{F}+\mathrm{e}$ & (a) & 50 \\
\hline $\mathrm{e}+\mathrm{CF} \rightarrow \mathrm{CF}^{+}+2 \mathrm{e}$ & (a) & 50 \\
\hline $\mathrm{e}+\mathrm{F}_{2} \rightarrow \mathrm{F}^{-}+\mathrm{F}$ & (a) & 51 \\
\hline $\mathrm{e}+\mathrm{F}_{2} \rightarrow 2 \mathrm{~F}+\mathrm{e}$ & (a) & 51 \\
\hline $\mathrm{e}+\mathrm{F}_{2} \rightarrow \mathrm{F}_{2}^{+}+2 \mathrm{e}$ & (a) & 51 \\
\hline $\mathrm{e}+\mathrm{F} \rightarrow \mathrm{F}^{*}+\mathrm{e}$ & (a) & 52 \\
\hline $\mathrm{e}+\mathrm{F} \rightarrow \mathrm{F}^{+}+2 \mathrm{e}$ & (a) & 52 \\
\hline $\mathrm{e}+\mathrm{F}^{*} \rightarrow \mathrm{F}+\mathrm{e}$ & (a) & 52 \\
\hline $\mathrm{e}+\mathrm{F}^{*} \rightarrow \mathrm{F}^{+}+2 \mathrm{e}$ & (a) & 52 \\
\hline $\mathrm{e}+\mathrm{CF}_{3}^{+} \rightarrow \mathrm{CF}_{2}+\mathrm{F}$ & (a) & 52 \\
\hline $\mathrm{e}+\mathrm{CF}_{2}^{+} \rightarrow \mathrm{CF}+\mathrm{F}$ & (a) & 52 \\
\hline $\mathrm{e}+\mathrm{F}_{2}^{+} \rightarrow 2 \mathrm{~F}$ & (a) & 52 \\
\hline $\mathrm{e}+\mathrm{F}^{+} \rightarrow \mathrm{F}$ & (a) & 52 \\
\hline $\mathrm{e}+\mathrm{O}_{2} \rightarrow \mathrm{O}_{2}^{+}+2 \mathrm{e}$ & (a) & 53 \\
\hline $\mathrm{e}+\mathrm{O}_{2} \rightarrow \mathrm{O}_{2}^{*}+\mathrm{e}$ & (a) & 53 \\
\hline $\mathrm{e}+\mathrm{O}_{2} \rightarrow 2 \mathrm{O}+\mathrm{e}$ & (a) & 53 \\
\hline $\mathrm{e}+\mathrm{O}_{2} \rightarrow \mathrm{O}^{*}+\mathrm{O}+\mathrm{e}$ & (a) & 53 \\
\hline $\mathrm{e}+\mathrm{O}_{2} \rightarrow \mathrm{O}+\mathrm{O}^{-}$ & (a) & 53 \\
\hline $\mathrm{e}+\mathrm{O}_{2} \rightarrow \mathrm{O}+\mathrm{O}^{+}+2 \mathrm{e}$ & (a) & 53 \\
\hline $\mathrm{e}+\mathrm{O}_{2}^{+} \rightarrow 2 \mathrm{O}$ & $1.2 \times 10^{-8} T_{e}^{-0.7}$ & 54 \\
\hline $\mathrm{e}+\mathrm{O}_{2}^{+} \rightarrow \mathrm{O}^{*}+\mathrm{O}$ & $8.88 \times 10^{-9} T_{e}^{-0.7}$ & 54 \\
\hline $\mathrm{e}+\mathrm{O}^{-} \rightarrow \mathrm{O}+2 \mathrm{e}$ & $1.95 \times 10^{-12} T_{e}^{-0.5} \exp \left[-3.4 / T_{e}\right]$ & 54 \\
\hline $\mathrm{e}+\mathrm{O} \rightarrow \mathrm{O}^{+}+2 \mathrm{e}$ & (a) & 55 \\
\hline $\mathrm{e}+\mathrm{O} \rightarrow \mathrm{O}^{*}+\mathrm{e}$ & (a) & 55 \\
\hline $\mathrm{e}+\mathrm{O}^{*} \rightarrow \mathrm{O}^{+}+2 \mathrm{e}$ & (a) & 55 \\
\hline $\mathrm{e}+\mathrm{O}^{*} \rightarrow \mathrm{O}+\mathrm{e}$ & (a) & 55 \\
\hline $\mathrm{e}+\mathrm{CO} \rightarrow \mathrm{C}+\mathrm{O}+\mathrm{e}$ & (a) & 56 \\
\hline $\mathrm{e}+\mathrm{CO} \rightarrow \mathrm{CO}^{+}+2 \mathrm{e}$ & (a) & 56 \\
\hline $\mathrm{e}+\mathrm{CO}_{2} \rightarrow \mathrm{CO}+\mathrm{O}^{-}$ & (a) & 57 \\
\hline $\mathrm{e}+\mathrm{CO}_{2} \rightarrow \mathrm{CO}_{2}^{+}+2 \mathrm{e}$ & (a) & 57 \\
\hline $\mathrm{e}+\mathrm{CO}_{2}^{+} \rightarrow \mathrm{CO}+\mathrm{O}$ & (a) & 57 \\
\hline $\mathrm{e}+\mathrm{COF}_{2} \rightarrow \mathrm{COF}+\mathrm{F}+\mathrm{e}$ & (a) & 49 \\
\hline
\end{tabular}

probability of 1 at the walls, and they return to the plasma as the corresponding neutrals. The sticking coefficients for $\mathrm{CF}_{3}$, $\mathrm{CF}_{2}, \mathrm{CF}, \mathrm{F}$, and $\mathrm{C}$ are set as $0.006,0.06,0.26,0.1$, and 0.6 , respectively, as obtained by molecular dynamics simulations. ${ }^{59}$ In addition, since the secondary electrons only have an important influence on the ionization, and induce transitions of the electron heating mode from $\alpha$ to $\gamma$ at high pressures and high voltages, ${ }^{1,60,61}$ secondary electron emission can be neglected in the model. The transport coefficients and elastic cross sections for the heavy species are calculated by using the Lennard-Jones parameters. ${ }^{62}$

\section{EXPERIMENTAL SETUP}

The experiments are carried out in a LAM 2300 Exelan Flex reactor, which is used for etching of $300 \mathrm{~mm}$ wafers. ${ }^{48}$ The schematic diagram of this cylindrical CCP reactor is shown in Fig. 2. The gas mixture is introduced in the reactor through a showerhead, which also serves as the top electrode. The wafer is placed on top of the bottom electrode, which is surrounded by a so-called "hot edge ring," made of pure Si. A quartz ring with a low dielectric constant (i.e., $\varepsilon / \varepsilon_{0}=3.9$ ) is placed out of the hot edge ring, in order to reduce the radio-frequency (rf) electric field near the wall. The bottom electrode is powered by rf sources (both SF and DF can be used), and the top electrode and all other surfaces in the reactor are grounded metal.

The substrates used for this experimental study are $300 \mathrm{~mm}$ silicon on insulator wafers with a $100 \mathrm{~nm}$ thick $\mathrm{SiO}_{2}$ layer and $200 \mathrm{~nm}$ polysilicon layer on top. By means of spectroscopic ellipsometry (KLA Tencor), the etch rate was determined by measuring the thickness of the amorphous top layer before and after the etch process.

\section{RESULTS AND DISCUSSION}

In this paper, we mainly focus on the effect of the LF power on the plasma properties, and especially on the plasma uniformity, in DF (HF and VHF) CCPs. However, first, the plasma composition is presented in Sec. IV A, to elucidate which are the important species in the plasma that are responsible for the etch rate in Sec. IV C. Subsequently, the plasma distributions obtained with and without Maxwell solver for a SF (HF and VHF) CCP are presented in Sec. IV B. From this section, it will be clear that the electromagnetic effects have an important influence on the plasma uniformity when the frequency is in the HF and VHF regime, which accordingly affects the etch process. Hence, in order to suppress the nonuniformity caused by the electromagnetic effects, a LF source is added to the discharge in Sec. IV C, and the influence of the LF power on the plasma uniformity will be investigated in detail by examining the measured etch rate and comparing it with the calculated fluxes of the important plasma species, in a wide range of LF powers.

\section{A. Plasma composition}

In order to obtain a general idea of the plasma composition, the calculated volume averaged densities of all species by using the full hybrid 2D model are shown in Fig. 3, for a $\mathrm{CF}_{4} / \mathrm{O}_{2}$ ratio of $0.9 / 0.1$ at 50 mTorr gas pressure and 100 sccm gas flow rate, when the discharge is sustained by a SF source, i.e., $27 \mathrm{MHz}$ at $300 \mathrm{~W}$. 
TABLE III. Heavy particle reactions included in the model.

\begin{tabular}{|c|c|c|}
\hline Reaction & Rate coefficient $\left(\mathrm{cm}^{3} / \mathrm{s}\right)$ & Reference \\
\hline $\mathrm{F}^{-}+\mathrm{CF}_{3} \rightarrow \mathrm{CF}_{4}+\mathrm{e}$ & $4.0 \times 10^{-10}$ & 54 \\
\hline $\mathrm{F}^{-}+\mathrm{CF}_{2} \rightarrow \mathrm{CF}_{3}+\mathrm{e}$ & $3.0 \times 10^{-10}$ & 54 \\
\hline $\mathrm{F}^{-}+\mathrm{CF} \rightarrow \mathrm{CF}_{2}+\mathrm{e}$ & $2.0 \times 10^{-10}$ & 54 \\
\hline $\mathrm{F}^{-}+\mathrm{C} \rightarrow \mathrm{CF}+\mathrm{e}$ & $1.0 \times 10^{-10}$ & 54 \\
\hline $\mathrm{F}^{-}+\mathrm{F} \rightarrow \mathrm{F}_{2}+\mathrm{e}$ & $1.0 \times 10^{-10}$ & 54 \\
\hline $\mathrm{F}^{-}+\mathrm{CF}_{3}^{+} \rightarrow \mathrm{CF}_{2}+\mathrm{F}_{2}$ & $8.7 \times 10^{-8}$ & 54 \\
\hline $\mathrm{F}^{-}+\mathrm{CF}_{3}^{+} \rightarrow \mathrm{CF}_{2}+2 \mathrm{~F}$ & $3.0 \times 10^{-7}$ & 54 \\
\hline $\mathrm{F}^{-}+\mathrm{CF}_{3}^{+} \rightarrow \mathrm{CF}_{3}+\mathrm{F}$ & $8.7 \times 10^{-7}$ & 54 \\
\hline $\mathrm{F}^{-}+\mathrm{CF}_{2}^{+} \rightarrow \mathrm{CF}+\mathrm{F}_{2}$ & $9.1 \times 10^{-8}$ & 54 \\
\hline $\mathrm{F}^{-}+\mathrm{CF}_{2}^{+} \rightarrow \mathrm{CF}_{2}+\mathrm{F}$ & $9.1 \times 10^{-8}$ & 54 \\
\hline $\mathrm{F}^{-}+\mathrm{CF}^{+} \rightarrow \mathrm{CF}+\mathrm{F}$ & $9.8 \times 10^{-8}$ & 54 \\
\hline $\mathrm{F}^{-}+\mathrm{CF}^{+} \rightarrow \mathrm{C}+2 \mathrm{~F}$ & $4.0 \times 10^{-7}$ & 54 \\
\hline $\mathrm{F}^{-}+\mathrm{F}_{2}^{+} \rightarrow \mathrm{F}+\mathrm{F}_{2}$ & $9.4 \times 10^{-8}$ & 54 \\
\hline $\mathrm{F}^{-}+\mathrm{F}^{+} \rightarrow 2 \mathrm{~F}$ & $3.1 \times 10^{-7}$ & 54 \\
\hline $\mathrm{CF}_{3}^{-}+\mathrm{CF}_{3}^{+} \rightarrow 2 \mathrm{CF}_{3}$ & $1.5 \times 10^{-7}$ & 54 \\
\hline $\mathrm{CF}_{3}^{-}+\mathrm{CF}_{2}^{+} \rightarrow \mathrm{CF}_{3}+\mathrm{CF}_{2}$ & $2.0 \times 10^{-7}$ & 54 \\
\hline $\mathrm{CF}_{3}^{-}+\mathrm{CF}^{+} \rightarrow \mathrm{CF}_{3}+\mathrm{CF}$ & $2.0 \times 10^{-7}$ & 54 \\
\hline $\mathrm{CF}_{3}^{-}+\mathrm{F}^{+} \rightarrow \mathrm{CF}_{3}+\mathrm{F}$ & $2.5 \times 10^{-7}$ & 54 \\
\hline $\mathrm{CF}_{3}^{-}+\mathrm{F}_{2}^{+} \rightarrow \mathrm{CF}_{3}+\mathrm{F}_{2}$ & $2.0 \times 10^{-7}$ & 54 \\
\hline $\mathrm{CF}_{2}^{+}+\mathrm{CF}_{4} \rightarrow \mathrm{CF}_{3}^{+}+\mathrm{CF}_{3}$ & $4.0 \times 10^{-10}$ & 54 \\
\hline $\mathrm{CF}_{2}^{+}+\mathrm{CF}_{3} \rightarrow \mathrm{CF}_{3}^{+}+\mathrm{CF}_{2}$ & $1.48 \times 10^{-9}$ & 54 \\
\hline $\mathrm{CF}_{2}^{+}+\mathrm{CF} \rightarrow \mathrm{CF}_{3}^{+}+\mathrm{C}$ & $2.06 \times 10^{-9}$ & 54 \\
\hline $\mathrm{CF}_{2}^{+}+\mathrm{C} \rightarrow \mathrm{CF}^{+}+\mathrm{CF}$ & $1.04 \times 10^{-9}$ & 54 \\
\hline $\mathrm{CF}^{+}+\mathrm{CF}_{4} \rightarrow \mathrm{CF}_{3}^{+}+\mathrm{CF}_{2}$ & $1.8 \times 10^{-10}$ & 54 \\
\hline $\mathrm{CF}^{+}+\mathrm{CF}_{3} \rightarrow \mathrm{CF}_{3}^{+}+\mathrm{CF}$ & $1.71 \times 10^{-9}$ & 54 \\
\hline $\mathrm{CF}^{+}+\mathrm{CF}_{2} \rightarrow \mathrm{CF}_{2}^{+}+\mathrm{CF}$ & $1.0 \times 10^{-9}$ & 54 \\
\hline $\mathrm{F}^{+}+\mathrm{CF}_{4} \rightarrow \mathrm{CF}_{3}^{+}+\mathrm{F}_{2}$ & $1.0 \times 10^{-9}$ & 54 \\
\hline $\mathrm{F}^{+}+\mathrm{CF}_{3} \rightarrow \mathrm{CF}_{2}^{+}+\mathrm{F}_{2}$ & $2.9 \times 10^{-9}$ & 54 \\
\hline $\mathrm{F}^{+}+\mathrm{CF}_{2} \rightarrow \mathrm{CF}^{+}+\mathrm{F}_{2}$ & $2.28 \times 10^{-9}$ & 54 \\
\hline $\mathrm{F}^{+}+\mathrm{F}_{2} \rightarrow \mathrm{F}_{2}^{+}+\mathrm{F}$ & $7.94 \times 10^{-10}$ & 54 \\
\hline $\mathrm{F}_{2}^{+}+\mathrm{CF}_{4} \rightarrow \mathrm{CF}_{3}^{+}+\mathrm{F}+\mathrm{F}_{2}$ & $1.0 \times 10^{-10}$ & 54 \\
\hline $\mathrm{F}_{2}^{+}+\mathrm{CF}_{3} \rightarrow \mathrm{CF}_{3}^{+}+2 \mathrm{~F}$ & $1.6 \times 10^{-9}$ & 54 \\
\hline $\mathrm{F}_{2}^{+}+\mathrm{CF}_{2} \rightarrow \mathrm{CF}_{2}^{+}+\mathrm{F}_{2}$ & $1.0 \times 10^{-9}$ & 54 \\
\hline $\mathrm{F}_{2}^{+}+\mathrm{CF}_{2} \rightarrow \mathrm{CF}_{3}^{+}+\mathrm{F}$ & $1.79 \times 10^{-9}$ & 54 \\
\hline $\mathrm{F}_{2}^{+}+\mathrm{CF} \rightarrow \mathrm{CF}_{2}^{+}+\mathrm{F}$ & $2.18 \times 10^{-9}$ & 54 \\
\hline $\mathrm{F}_{2}^{+}+\mathrm{C} \rightarrow \mathrm{CF}^{+}+\mathrm{F}$ & $1.04 \times 10^{-9}$ & 54 \\
\hline $\mathrm{CF}_{3}^{-}+\mathrm{F} \rightarrow \mathrm{CF}_{3}+\mathrm{F}^{-}$ & $5.0 \times 10^{-8}$ & 54 \\
\hline $\mathrm{O}^{+}+\mathrm{O}_{2} \rightarrow \mathrm{O}_{2}^{+}+\mathrm{O}$ & $2.0 \times 10^{-11}$ & 46 \\
\hline $\mathrm{O}^{-}+\mathrm{O}^{+} \rightarrow 2 \mathrm{O}$ & $2.7 \times 10^{-7}[T / 298]^{-0.5}$ & 46 \\
\hline $\mathrm{O}^{-}+\mathrm{O}_{2}^{+} \rightarrow \mathrm{O}+\mathrm{O}_{2}$ & $2.0 \times 10^{-7}[T / 298]^{-0.5}$ & 46 \\
\hline $\mathrm{O}^{-}+\mathrm{O}_{2}^{+} \rightarrow 3 \mathrm{O}$ & $1.0 \times 10^{-7}$ & 46 \\
\hline $\mathrm{O}^{-}+\mathrm{O} \rightarrow \mathrm{O}_{2}+\mathrm{e}$ & $2.0 \times 10^{-10}$ & 46 \\
\hline $\mathrm{O}^{*}+\mathrm{O} \rightarrow 2 \mathrm{O}$ & $8.0 \times 10^{-12}$ & 46 \\
\hline $\mathrm{O}^{*}+\mathrm{O}_{2} \rightarrow \mathrm{O}+\mathrm{O}_{2}$ & $2.56 \times 10^{-11} \exp [67.0 / T]$ & 46 \\
\hline $\mathrm{O}^{*}+\mathrm{CF}_{4} \rightarrow \mathrm{O}+\mathrm{CF}_{4}$ & $1.8 \times 10^{-13}$ & 54 \\
\hline $\mathrm{O}^{*}+\mathrm{CF}_{3} \rightarrow \mathrm{COF}_{2}+\mathrm{F}$ & $3.1 \times 10^{-11}$ & 54 \\
\hline $\mathrm{O}^{*}+\mathrm{CF}_{2} \rightarrow \mathrm{COF}+\mathrm{F}$ & $1.4 \times 10^{-11}$ & 54 \\
\hline $\mathrm{O}^{*}+\mathrm{CF}_{2} \rightarrow \mathrm{CO}+2 \mathrm{~F}$ & $4.0 \times 10^{-12}$ & 54 \\
\hline $\mathrm{O}^{*}+\mathrm{CF} \rightarrow \mathrm{CO}+\mathrm{F}$ & $2.0 \times 10^{-11}$ & 54 \\
\hline $\mathrm{O}^{*}+\mathrm{COF}_{2} \rightarrow \mathrm{O}+\mathrm{COF}_{2}$ & $5.3 \times 10^{-11}$ & 54 \\
\hline $\mathrm{O}^{*}+\mathrm{COF}_{2} \rightarrow \mathrm{F}_{2}+\mathrm{CO}_{2}$ & $2.1 \times 10^{-11}$ & 54 \\
\hline $\mathrm{O}^{*}+\mathrm{COF} \rightarrow \mathrm{CO}_{2}+\mathrm{F}$ & $9.3 \times 10^{-11}$ & 54 \\
\hline $\mathrm{O}^{*}+\mathrm{FO} \rightarrow \mathrm{O}_{2}+\mathrm{F}$ & $5.0 \times 10^{-11}$ & 54 \\
\hline $\mathrm{O}+\mathrm{FO} \rightarrow \mathrm{O}_{2}+\mathrm{F}$ & $2.7 \times 10^{-11}$ & 54 \\
\hline $\mathrm{C}+\mathrm{O}_{2} \rightarrow \mathrm{CO}+\mathrm{O}$ & $1.6 \times 10^{-11}$ & 54 \\
\hline $\mathrm{COF}+\mathrm{CF}_{2} \rightarrow \mathrm{CF}_{3}+\mathrm{CO}$ & $3.0 \times 10^{-13}$ & 54 \\
\hline $\mathrm{COF}+\mathrm{CF}_{2} \rightarrow \mathrm{COF}_{2}+\mathrm{CF}$ & $3.0 \times 10^{-13}$ & 54 \\
\hline $\mathrm{COF}+\mathrm{CF}_{3} \rightarrow \mathrm{CF}_{4}+\mathrm{CO}$ & $1.0 \times 10^{-11}$ & 54 \\
\hline $\mathrm{COF}+\mathrm{CF}_{3} \rightarrow \mathrm{COF}_{2}+\mathrm{CF}_{2}$ & $1.0 \times 10^{-11}$ & 54 \\
\hline
\end{tabular}

TABLE III. (Continued)

\begin{tabular}{|c|c|c|}
\hline Reaction & Rate coefficient $\left(\mathrm{cm}^{3} / \mathrm{s}\right)$ & Reference \\
\hline $\mathrm{COF}+\mathrm{COF} \rightarrow \mathrm{COF}_{2}+\mathrm{CO}$ & $1.0 \times 10^{-11}$ & 54 \\
\hline $\mathrm{O}+\mathrm{CF} \rightarrow \mathrm{CO}+\mathrm{F}$ & $6.6 \times 10^{-11}$ & 54 \\
\hline $\mathrm{O}+\mathrm{CF}_{2} \rightarrow \mathrm{COF}+\mathrm{F}$ & $3.1 \times 10^{-11}$ & 54 \\
\hline $\mathrm{O}+\mathrm{CF}_{2} \rightarrow \mathrm{CO}+2 \mathrm{~F}$ & $4.0 \times 10^{-12}$ & 54 \\
\hline $\mathrm{O}+\mathrm{CF}_{3} \rightarrow \mathrm{COF}_{2}+\mathrm{F}$ & $3.3 \times 10^{-11}$ & 54 \\
\hline $\mathrm{O}+\mathrm{COF} \rightarrow \mathrm{CO}_{2}+\mathrm{F}$ & $9.3 \times 10^{-11}$ & 54 \\
\hline $\mathrm{O}_{2}+\mathrm{CF} \rightarrow \mathrm{COF}+\mathrm{O}$ & $3.3 \times 10^{-11}$ & 54 \\
\hline $\mathrm{CO}^{+}+\mathrm{O} \rightarrow \mathrm{O}^{+}+\mathrm{CO}$ & $1.4 \times 10^{-10}$ & 54 \\
\hline $\mathrm{CO}^{+}+\mathrm{O}_{2} \rightarrow \mathrm{O}_{2}^{+}+\mathrm{CO}$ & $1.2 \times 10^{-10}$ & 54 \\
\hline $\mathrm{CO}^{+}+\mathrm{CF}_{4} \rightarrow \mathrm{CF}_{3}^{+}+\mathrm{COF}$ & $7.0 \times 10^{-10}$ & 54 \\
\hline $\mathrm{CO}^{+}+\mathrm{CF}_{3} \rightarrow \mathrm{CF}_{2}^{+}+\mathrm{COF}$ & $7.0 \times 10^{-10}$ & 54 \\
\hline $\mathrm{CO}^{+}+\mathrm{CF}_{2} \rightarrow \mathrm{CF}^{+}+\mathrm{COF}$ & $7.0 \times 10^{-10}$ & 54 \\
\hline $\mathrm{F}^{+}+\mathrm{O} \rightarrow \mathrm{O}^{+}+\mathrm{F}$ & $1.0 \times 10^{-10}$ & 54 \\
\hline $\mathrm{F}^{+}+\mathrm{O}_{2} \rightarrow \mathrm{O}_{2}^{+}+\mathrm{F}$ & $7.14 \times 10^{-10}$ & 54 \\
\hline $\mathrm{F}^{+}+\mathrm{O}_{2} \rightarrow \mathrm{O}^{+}+\mathrm{FO}$ & $5.04 \times 10^{-11}$ & 54 \\
\hline $\mathrm{O}^{+}+\mathrm{CF}_{4} \rightarrow \mathrm{CF}_{3}^{+}+\mathrm{FO}$ & $1.4 \times 10^{-9}$ & 54 \\
\hline $\mathrm{F}^{-}+\mathrm{O} \rightarrow \mathrm{F}+\mathrm{O}+\mathrm{e}$ & $1.0 \times 10^{-10}$ & 54 \\
\hline $\mathrm{CF}_{3}^{-}+\mathrm{O}_{2}^{+} \rightarrow \mathrm{CF}_{3}+\mathrm{O}_{2}$ & $2.0 \times 10^{-7}$ & 54 \\
\hline $\mathrm{CF}_{3}^{-}+\mathrm{O}^{+} \rightarrow \mathrm{CF}_{3}+\mathrm{O}$ & $2.5 \times 10^{-7}$ & 54 \\
\hline $\mathrm{CF}_{3}^{-}+\mathrm{CO}^{+} \rightarrow \mathrm{CF}_{3}+\mathrm{CO}$ & $2.0 \times 10^{-7}$ & 54 \\
\hline $\mathrm{CF}_{3}^{-}+\mathrm{CO}_{2}^{+} \rightarrow \mathrm{CF}_{3}+\mathrm{CO}_{2}$ & $2.0 \times 10^{-7}$ & $54^{\mathrm{a}}$ \\
\hline $\mathrm{F}^{-}+\mathrm{O}_{2}^{+} \rightarrow \mathrm{F}+\mathrm{O}_{2}$ & $3.0 \times 10^{-7}$ & 54 \\
\hline $\mathrm{F}^{-}+\mathrm{CO}^{+} \rightarrow \mathrm{F}+\mathrm{CO}$ & $3.0 \times 10^{-7}$ & 54 \\
\hline $\mathrm{F}^{-}+\mathrm{CO}_{2}^{+} \rightarrow \mathrm{F}+\mathrm{CO}_{2}$ & $3.0 \times 10^{-7}$ & $54^{\mathrm{a}}$ \\
\hline $\mathrm{F}^{-}+\mathrm{O}^{+} \rightarrow \mathrm{F}+\mathrm{O}$ & $3.0 \times 10^{-7}$ & 54 \\
\hline $\mathrm{O}^{-}+\mathrm{CF}_{3}^{+} \rightarrow \mathrm{O}+\mathrm{CF}_{3}$ & $2.0 \times 10^{-7}$ & 54 \\
\hline $\mathrm{O}^{-}+\mathrm{CF}_{2}^{+} \rightarrow \mathrm{O}+\mathrm{CF}_{2}$ & $2.0 \times 10^{-7}$ & 54 \\
\hline $\mathrm{O}^{-}+\mathrm{CF}^{+} \rightarrow \mathrm{O}+\mathrm{CF}$ & $2.0 \times 10^{-7}$ & 54 \\
\hline $\mathrm{O}^{-}+\mathrm{CF}^{+} \rightarrow \mathrm{O}+\mathrm{CF}$ & $2.0 \times 10^{-7}$ & 54 \\
\hline $\mathrm{O}^{-}+\mathrm{F}^{+} \rightarrow \mathrm{O}+\mathrm{F}$ & $2.0 \times 10^{-7}$ & 54 \\
\hline $\mathrm{O}^{-}+\mathrm{F}_{2}^{+} \rightarrow \mathrm{O}+\mathrm{F}_{2}$ & $1.5 \times 10^{-7}$ & 54 \\
\hline $\mathrm{O}^{-}+\mathrm{CO}^{+} \rightarrow \mathrm{O}+\mathrm{CO}$ & $2.0 \times 10^{-7}$ & 54 \\
\hline $\mathrm{O}^{-}+\mathrm{CO}_{2}^{+} \rightarrow \mathrm{O}+\mathrm{CO}_{2}$ & $2.0 \times 10^{-7}$ & $54^{\mathrm{a}}$ \\
\hline
\end{tabular}

${ }^{\mathrm{a}}$ Estimated.

It is clear that $\mathrm{CF}_{4}$ is the dominant species in the discharge, and its density (i.e., $1.2 \times 10^{15} \mathrm{~cm}^{-3}$ ) is almost one order of magnitude higher than for $\mathrm{O}_{2}$ (i.e., $1.3 \times 10^{14}$ $\mathrm{cm}^{-3}$ ), which reflects the gas ratio. $\mathrm{CF}_{4}$ is mainly lost by electron impact dissociation, dissociative ionization, and dissociative attachment. The first process is most important, and gives rise to a high density of $\mathrm{CF}_{3}$ radicals (i.e., $4.7 \times 10^{13} \mathrm{~cm}^{-3}$ ) and $\mathrm{F}$ atoms (i.e., $9.8 \times 10^{12} \mathrm{~cm}^{-3}$ ). Other important plasma species are $\mathrm{COF}_{2}$ and $\mathrm{CO}_{2}$, whose densities are in the same order of magnitude as the $\mathrm{CF}_{3}$ density. This can be explained by the high rate coefficient between $\mathrm{CF}_{3}$ and $\mathrm{O}$ (the main reactants for the formation of $\mathrm{COF}_{2}$ ), as well as their high densities. On the other hand, a large amount of $\mathrm{COF}_{2}$ is lost by the collision with $\mathrm{O}^{*}$, which accordingly gives rise to a high density of $\mathrm{CO}_{2}$. Furthermore, also the $\mathrm{F}_{2}$ and $\mathrm{CO}$ densities are rather high. $\mathrm{F}_{2}$ is formed by electron impact dissociation of $\mathrm{CF}_{4}$ and the recombination between $\mathrm{F}^{-}$and $\mathrm{CF}_{3}^{+}$ions, while $\mathrm{CO}$ is mainly created out of the reactions $\mathrm{O}+\mathrm{CF}_{2} \rightarrow \mathrm{CO}+2 \mathrm{~F}$ and $\mathrm{C}+\mathrm{O}_{2} \rightarrow \mathrm{CO}+\mathrm{O}$.

The densities of the charged species are several orders of magnitude lower than for their neutral counterparts, as is 


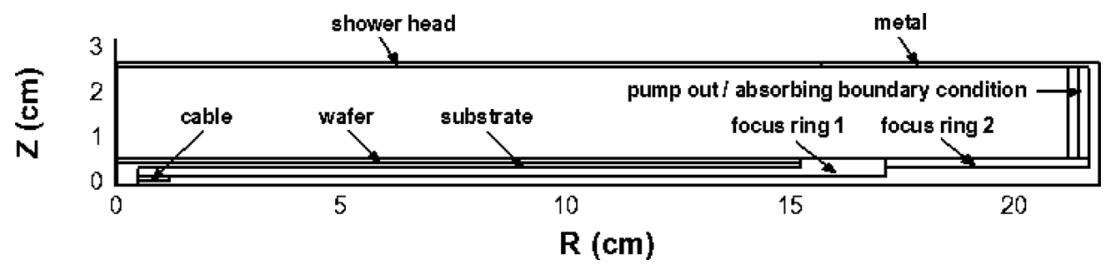

FIG. 2. Schematic picture of the cylindrical capacitively coupled plasma reactor.

obvious from Fig. 3. The $\mathrm{CF}_{3}^{+}$ions are the most abundant positive ions present in the plasma, followed by $\mathrm{O}_{2}^{+}$, which are mostly formed from electron impact ionization from the background gases. It is interesting to note that although the $\mathrm{CF}_{4}$ density is almost ten times higher than that of $\mathrm{O}_{2}$, the $\mathrm{O}_{2}^{+}$density (i.e., $7.9 \times 10^{9} \mathrm{~cm}^{-3}$ ) is only a factor of 4 lower than the $\mathrm{CF}_{3}^{+}$density (i.e., $3.2 \times 10^{10} \mathrm{~cm}^{-3}$ ). This can be attributed to the higher ionization threshold for $\mathrm{CF}_{4}$ than for $\mathrm{O}_{2}$ (i.e., $16.25 \mathrm{eV}$ for $\mathrm{CF}_{4}$ and $12 \mathrm{eV}$ for $\mathrm{O}_{2}$ ), as well as the lower cross section when the electron temperature is lower than about $30 \mathrm{eV} .^{49,53}$ Moreover, the $\mathrm{CO}_{2}^{+}$ions also have a high density; they are mainly generated by electron impact ionization of $\mathrm{CO}_{2}$.

Due to the electronegativity of $\mathrm{CF}_{4}$ and $\mathrm{O}_{2}$, there exist a large amount of negative ions in the plasma. The dominant negative ion species is $\mathrm{F}^{-}$, whose density is about 20 times higher than for $\mathrm{CF}_{3}^{-}$(i.e., $1.2 \times 10^{10} \mathrm{~cm}^{-3}$ vs $5.4 \times 10^{8}$ $\mathrm{cm}^{-3}$ ). This is because the cross section for dissociative attachment of $\mathrm{CF}_{4}$ for $\mathrm{F}^{-}$production is five to six times higher than that for $\mathrm{CF}_{3}^{-}$production, ${ }^{63}$ and moreover the $\mathrm{F}^{-}$ ions can also be produced by electron impact dissociative attachment of $\mathrm{F}_{2}$ and $\mathrm{CF}_{3}$. The $\mathrm{O}^{-}$ions are mainly formed by electron impact dissociative attachment of $\mathrm{O}_{2}$, and their density is about two times lower than for $\mathrm{F}^{-}$(i.e., $5.6 \times 10^{9}$ $\mathrm{cm}^{-3}$ ). Hence, the $\mathrm{O}^{-} / \mathrm{F}^{-}$ratio is much higher than the $\mathrm{O}_{2} /$ $\mathrm{CF}_{4}$ ratio, which can be attributed to the higher rate coefficient for the recombination between $\mathrm{F}^{-}$and $\mathrm{CF}_{3}^{+}$ions. Nevertheless, in spite of the large abundance of negative ions, the electrons are still the major negative charge carriers at this condition. Note that these results apply to a $\mathrm{CF}_{4} / \mathrm{O}_{2}$ ratio of 90/10; when this ratio changes, the relative composition changes accordingly. However, in Secs. IV B and IV C, we also keep the $\mathrm{CF}_{4} / \mathrm{O}_{2}$ ratio fixed at 90/10. Moreover, the results are presented for a SF discharge at $27 \mathrm{MHz}$, but when

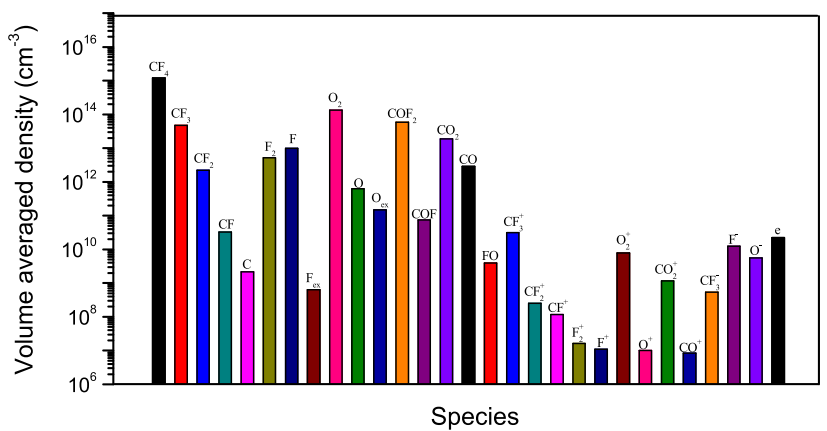

FIG. 3. (Color online) Volume averaged densities of all species in a $\mathrm{CF}_{4} / \mathrm{O}_{2}$ discharge sustained at $27 \mathrm{MHz}$ and $300 \mathrm{~W}, \mathrm{a} \mathrm{CF}_{4} / \mathrm{O}_{2}$ ratio of $90 / 10,50$ $\mathrm{mTorr}$ gas pressure, and a gas flow rate of $100 \mathrm{sccm}$. the discharge is sustained by a SF source of $60 \mathrm{MHz}$ or by DF sources (i.e., $27+2$ and $60+2 \mathrm{MHz}$ ), although the absolute values of the volume averaged densities of all species might be a bit different due to different discharge conditions, the plasma composition in general is very similar (hence it is not shown here).

\section{B. Electromagnetic effects}

In this section, we investigate the influence of the electromagnetic effects on the plasma properties, by examining the plasma density distributions obtained in the models with and without Maxwell solver, for a SF discharge at both 27 and $60 \mathrm{MHz}$. By solving Maxwell's equation, the effects resulting from wave penetration into the plasma are included, which gives rise to a different distribution of the electric field, and therefore, it affects the plasma behavior. ${ }^{31}$

The electron densities calculated without and with Maxwell solver at $27 \mathrm{MHz}$ show striking differences, as are apparent from Figs. 4(a) and 4(b). When the Maxwell solver is applied, a more uniform electron density distribution is observed with higher absolute values than in the case without Maxwell solver (i.e., maximum of about $3.2 \times 10^{10} \mathrm{~cm}^{-3}$ vs $\left.1.8 \times 10^{10} \mathrm{~cm}^{-3}\right)$. This can be explained because the axial inductive electric field has a maximum in the center, which enhances the electron heating and therefore increases the electron density in the center [see Fig. 10(b)]. This difference indicates that the inductive electric field has an important influence on the plasma properties, although the frequency $(27 \mathrm{MHz})$ is not yet in the VHF regime. Besides, the magnetic Reynold's number $\mathrm{R}_{\mathrm{m}}=\mu_{0} \mathrm{e}^{2} \mathrm{n}_{\mathrm{e}} \mathrm{L}_{0}^{2} / \mathrm{m}_{\mathrm{e}}$ can be used as a measure of the skin effect, where $L_{0}$ is the characteristic length scale. When the Maxwell solver is applied at $27 \mathrm{MHz}, \mathrm{R}_{\mathrm{m}}=36.5 \gg 1$ (volume averaged $\mathrm{n}_{\mathrm{e}} \sim 2.156 \times 10^{10} \mathrm{~cm}^{-3}$ ), and the skin effect has a significant influence on the plasma distribution, as was also reported in Ref. 64.

The higher electron density in Fig. 4(b) also leads to a higher $\mathrm{CF}_{4}$ ionization rate, and results in a higher $\mathrm{CF}_{3}^{+}$density, as is clear by comparing Figs. 4(c) and 4(d). Indeed, when the Maxwell solver is included [Fig. 4(d)], the maximum $\mathrm{CF}_{3}^{+}$density rises from $5.5 \times 10^{10}$ to $7.0 \times 10^{10} \mathrm{~cm}^{-3}$. The $\mathrm{CF}_{3}^{+}$density has a maximum near the edge of the bottom electrode in both cases, i.e., with and without Maxwell solver. Since $\mathrm{CF}_{3}^{+}$is mainly produced by electron impact ionization of $\mathrm{CF}_{4}$, the edge-peaked $\mathrm{CF}_{3}^{+}$density distribution reflects the higher ionization rate at the radial edge due to the higher electron temperature there. 

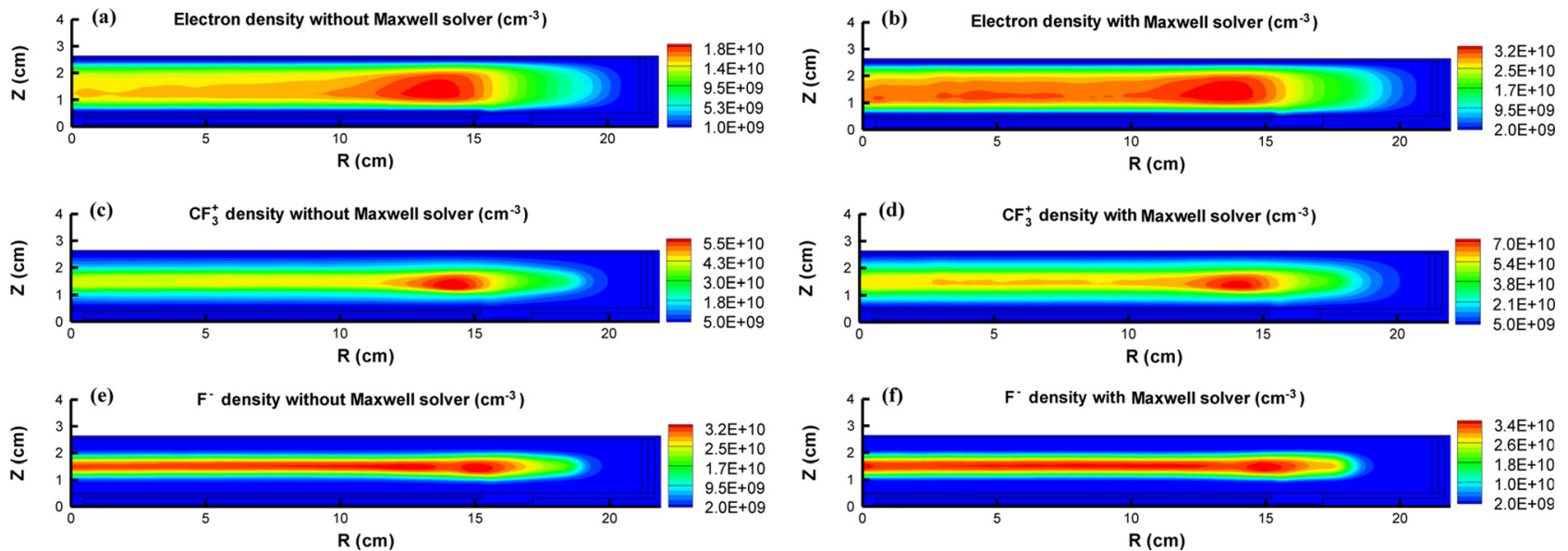

FIG. 4. (Color online) Distributions of the electron density, $\mathrm{CF}_{3}^{+}$density, and $\mathrm{F}^{-}$density calculated in the models without (left column) and with (right column) Maxwell solver, in a SF $\mathrm{CF}_{4} / \mathrm{O}_{2}$ discharge sustained at $27 \mathrm{MHz}$ and $300 \mathrm{~W}$. The other conditions are the same as in Fig. 3. Note the different color scales in the figures without and with Maxwell solver, which was necessary to clearly illustrate the different profiles.

The $\mathrm{F}^{-}$density profiles are shown in Figs. 4(e) and 4(f). It is clear that the electromagnetic effects have no significant influence on the shape of the $\mathrm{F}^{-}$density, i.e., the $\mathrm{F}^{-}$ions are confined to the electronegative core of the plasma with a uniform distribution along the radial direction. Besides, the maximum of the $\mathrm{F}^{-}$density increases only slightly from $3.2 \times 10^{10}$ to $3.4 \times 10^{10} \mathrm{~cm}^{-3}$ when the Maxwell solver is included, due to the higher electron impact dissociative attachment caused by the electromagnetic effects.

When the frequency increases to $60 \mathrm{MHz}$ (see Fig. 5), the distributions of the electron density are again strikingly different, both in shape and especially in absolute values, when the electromagnetic effects are taken into account. Indeed, the electron density calculated in the model combined with Maxwell solver [Fig. 5(b)] is higher and less uniform than that obtained in the case without electromagnetic effects [Fig. 5(a)]. Note that this trend is opposite from the behavior at $27 \mathrm{MHz}$, where the electron density profile was more uniform when the electromagnetic effects were taken into account.

By comparing Figs. 4(b) and 5(b), it is clear that the offaxis peak of the electron density becomes more evident as the frequency increases, which has also been observed by Yang and Kushner in $\mathrm{Ar} / \mathrm{CF}_{4}$ plasmas. ${ }^{31}$ Indeed, as the frequency increases from 27 to $60 \mathrm{MHz}$, the magnetic Reynold's number in the case with Maxwell solver varies from 36.5 to 42.6 when the volume averaged electron density increases to $2.517 \times 10^{10} \mathrm{~cm}^{-3}$. This indicates that the skin effect becomes even more dominant under this condition, and the off-axis maximum is attributed to the dominant peak of the radial inductive electric field near the radial edge. ${ }^{27,64}$ In addition, the electron density profile is different from that observed in pure Ar discharges, which shows two maxima, i.e., in the center and near the edge, and this is caused by the electronegativity of $\mathrm{CF}_{4}$ and $\mathrm{O}_{2}{ }^{31}$

The $\mathrm{CF}_{3}^{+}$density profile is not so much determined by the electromagnetic effects, as is clear from comparing Figs. 5(c) and 5(d); only its absolute value increases when including the electromagnetic effects, similar to the case of 27
MHz. However, the electromagnetic effects on the $\mathrm{F}^{-}$density are a bit more pronounced, as shown in Figs. 5(e) and 5(f). In the case without Maxwell solver, the $\mathrm{F}^{-}$density has a uniform distribution along the radial direction, which is similar to that observed at $27 \mathrm{MHz}$ [cf. Figs. 4(e) and 4(f)], except for a higher value. However, when the Maxwell solver is included, the uniformity becomes worse and the maximum of the $\mathrm{F}^{-}$density appears at the radial edge of the bottom electrode, which indicates that the electromagnetic effects have a more pronounced influence on the plasma characteristics at $60 \mathrm{MHz}$.

Radial distributions of the power deposition along the reactor centerline calculated in the models without and with Maxwell solver at 27 and $60 \mathrm{MHz}$ are shown in Fig. 6. It is clear that the power density is characterized by an edgepeaked profile under all the selected discharge conditions due to the skin effect, as mentioned above. When the Maxwell solver is included, the power deposition is higher than in the case without Maxwell solver both at 27 and 60 $\mathrm{MHz}$, due to the more efficient heating mechanisms caused by the electromagnetic field. Moreover, when the frequency is higher, i.e., $60 \mathrm{MHz}$, the difference between the power densities calculated in the models without and with Maxwell solver becomes more obvious than at $27 \mathrm{MHz}$, especially at the radial edge. This can be explained because the electromagnetic effects become more significant at $60 \mathrm{MHz}$.

Indeed, when the electromagnetic effects are included, the electrons are heated by both the electrostatic field and the electromagnetic field. This indicates that the electrodynamics is influenced by the electromagnetic effects, and so is the plasma chemistry. The rate coefficients of electron impact dissociative ionization, dissociation, and dissociative attachment of $\mathrm{CF}_{4}$ calculated without and with Maxwell solver at 27 and $60 \mathrm{MHz}$ are shown in Fig. 7. It is clear that when the Maxwell solver is included at $27 \mathrm{MHz}$, the rate coefficient of electron impact dissociative ionization of $\mathrm{CF}_{4}$ increases from $9.09 \times 10^{-11}$ to $1.01 \times 10^{-10} \mathrm{~cm}^{3} \mathrm{~s}^{-1}$, due to the higher electron temperature caused by the electromagnetic effects. When the frequency is $60 \mathrm{MHz}$, the ionization 

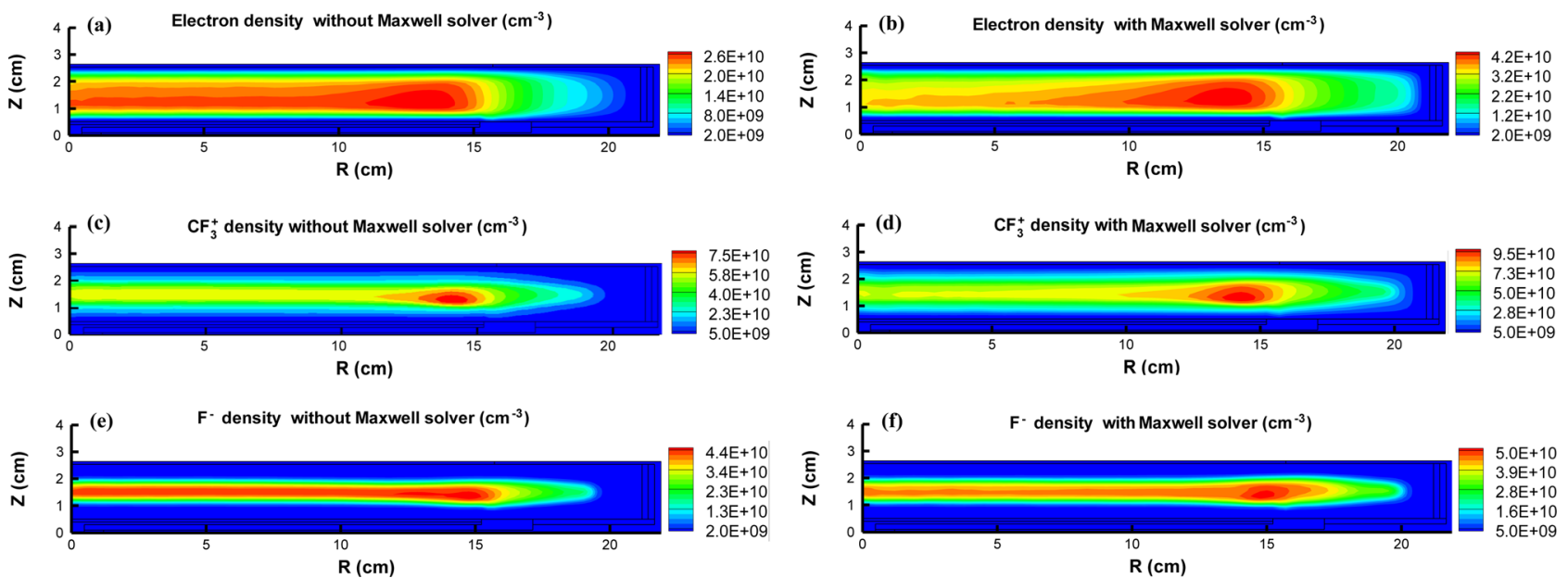

FIG. 5. (Color online) Distributions of the electron density, $\mathrm{CF}_{3}^{+}$density, and $\mathrm{F}^{-}$density calculated in the models without (left column) and with (right column) Maxwell solver, in a $\mathrm{SF} \mathrm{CF}_{4} / \mathrm{O}_{2}$ discharge sustained at $60 \mathrm{MHz}$ and $300 \mathrm{~W}$. The other conditions are the same as in Fig. 3. Note the different color scales in the figures without and with Maxwell solver, which was necessary to clearly illustrate the different profiles.

rate coefficient increases by about $20 \%$ (i.e., around $1.03 \times 10^{-10} \mathrm{~cm}^{3} \mathrm{~s}^{-1}$ in the case without Maxwell solver, and around $1.24 \times 10^{-10} \mathrm{~cm}^{3} \mathrm{~s}^{-1}$ in the case with Maxwell solver), which indicates that the electromagnetic effects become more pronounced at higher frequencies. However, the rate coefficients of electron impact dissociation and dissociative attachment of $\mathrm{CF}_{4}$ become lower when the Maxwell solver is employed both at 27 and $60 \mathrm{MHz}$. The different behavior of the rate coefficients for electron impact dissociation and dissociative attachment can be attributed to the lower threshold energy of these processes. Indeed, when the electromagnetic effects are taken into account, the electrons absorb more power (see Fig. 6), and the number of electrons with higher energy becomes larger, and this accordingly increases the ionization rate coefficient, while it reduces the dissociation and attachment rate coefficients.

Figure 8 shows the radial distributions of the $\mathrm{F}$ atom flux, the $\mathrm{O}$ atom flux, the $\mathrm{CF}_{2}$ radical flux, and the total positive ion flux above the bottom electrode, calculated both at 27

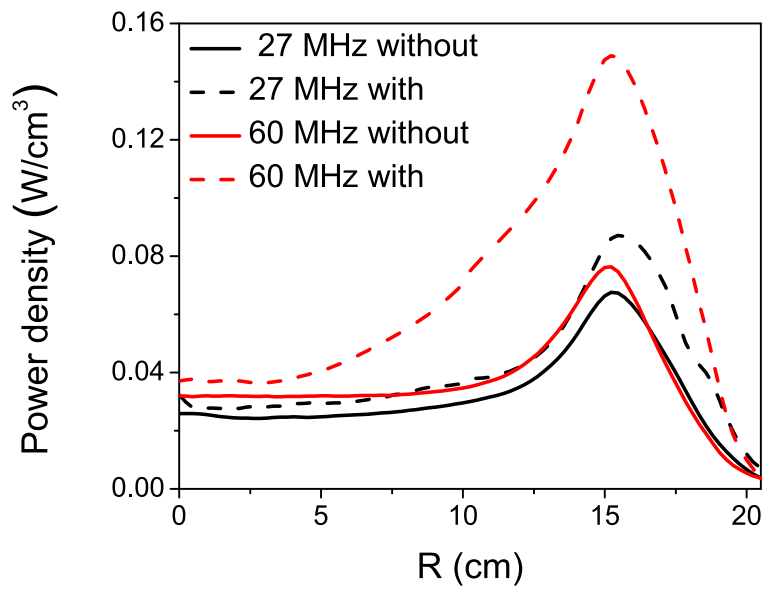

FIG. 6. (Color online) Radial distributions of the power deposition along the reactor centerline calculated in the models without and with Maxwell solver, in a $\mathrm{CF}_{4} / \mathrm{O}_{2}$ discharge sustained at 27 and $60 \mathrm{MHz}$, and $300 \mathrm{~W}$. The other conditions are also the same as in Fig. 3. and $60 \mathrm{MHz}$. We selected these species, since they contribute most to the etch process, as will be illustrated below. In general, the profiles of the fluxes obtained with and without Maxwell solver exhibit a similar shape (except for the $\mathrm{O}$ atom flux at $60 \mathrm{MHz}$ ), but the absolute values are higher when the electromagnetic effects are taken into account, at both 27 and $60 \mathrm{MHz}$. This is because the electrons are now heated not only by the electrostatic field, but also by the inductive electric field, which gives rise to a higher electron density, and accordingly it increases the electron impact reaction rates and hence the production of reactive species.

When comparing the effect of the frequency, it is clear that all the fluxes increase with higher frequency. This is caused by the more efficient electron heating and less power dissipation by ion acceleration. ${ }^{1}$ Moreover, the $\mathrm{F}$ atom flux profile [Fig. 8(a)] shifts from a rather uniform profile at 27 $\mathrm{MHz}$ to a broad maximum in the center at $60 \mathrm{MHz}$. This is because at $27 \mathrm{MHz}$, although a large amount of $\mathrm{F}$ atoms is lost by pumping at the radial edge, ${ }^{65}$ more $\mathrm{F}$ atoms are generated in this region due to the higher electron temperature caused by the radial electric field. However, at $60 \mathrm{MHz}$, the

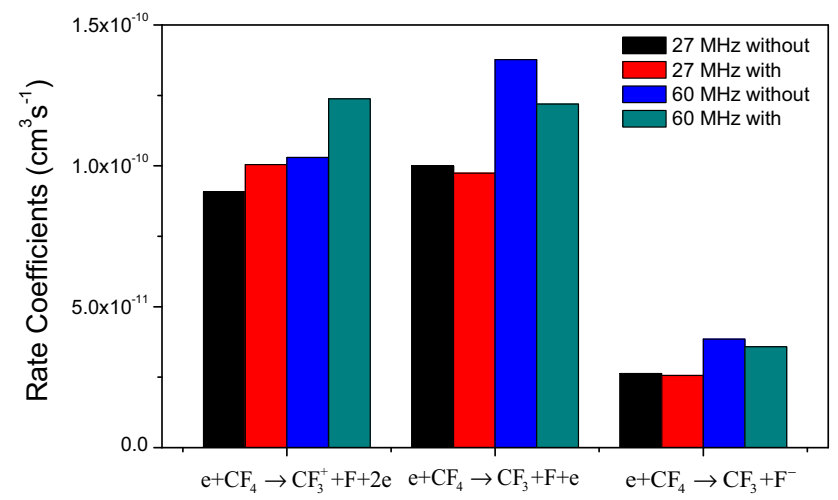

FIG. 7. (Color online) Rate coefficients of electron impact dissociative ionization, dissociation and dissociative attachment of $\mathrm{CF}_{4}$ calculated in the models without and with Maxwell solver, in a $\mathrm{CF}_{4} / \mathrm{O}_{2}$ discharge sustained at 27 and $60 \mathrm{MHz}$, and $300 \mathrm{~W}$. The other conditions are also the same as in Fig. 3. 

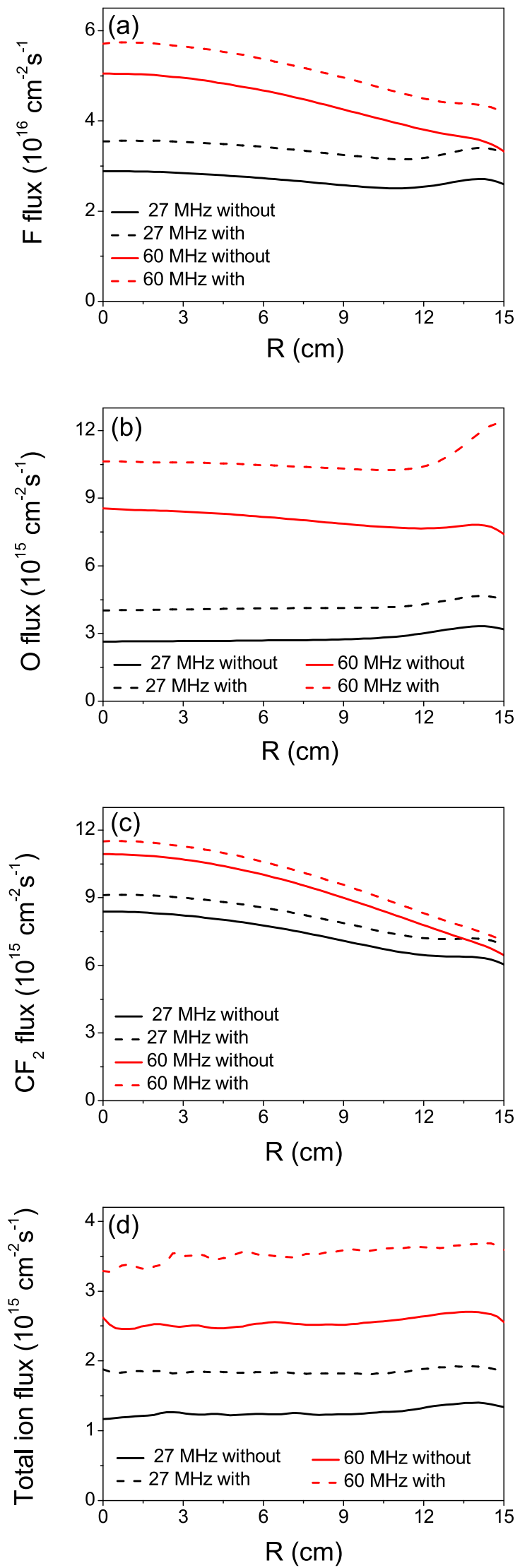

FIG. 8. (Color online) Radial distributions of the $\mathrm{F}$ atom flux (a), O atom flux (b), $\mathrm{CF}_{2}$ radical flux (c), and total positive ion flux (d) at the bottom electrode calculated in the models without and with Maxwell solver, in a $\mathrm{CF}_{4} / \mathrm{O}_{2}$ discharge sustained at 27 and $60 \mathrm{MHz}$, and $300 \mathrm{~W}$. The other conditions are also the same as in Fig. 3. loss due to the pumping has a dominant influence on the $\mathrm{F}$ atom flux distribution. The $\mathrm{O}$ atom flux [Fig. 8(b)] is uniform along the radial direction in the bulk region, but with a slightly higher value near the radial edge at $27 \mathrm{MHz}$, due to the higher electron density there. When the frequency increases to $60 \mathrm{MHz}$, the $\mathrm{O}$ atom flux calculated without Maxwell solver is characterized by a rather uniform profile, which is slightly higher in the center, but when the electromagnetic effects are taken into account, the $\mathrm{O}$ atom flux near the radial edge exhibits a significant increase from $7.8 \times 10^{15}$ to $1.2 \times 10^{16} \mathrm{~cm}^{-2} \mathrm{~s}^{-1}$. Thus, the $\mathrm{O}$ atom flux is characterized by a clear off-axis peak under this condition. The $\mathrm{CF}_{2}$ radical flux [Fig. 8(c)] exhibits a broad maximum in the center at $27 \mathrm{MHz}$, and this becomes even more pronounced as the frequency increases to $60 \mathrm{MHz}$. Finally, the total positive ion fluxes calculated with and without Maxwell solver at 27 and $60 \mathrm{MHz}$, presented in Fig. 8(d), exhibit similar profiles in all cases, except again for a remarkable increase in the magnitude of the fluxes, both at 27 and $60 \mathrm{MHz}$, due to the significant power deposition caused by the inductive electric field, as mentioned above.

In summary, it can be concluded that the calculated plasma species densities and their fluxes above the bottom electrode are influenced by the electromagnetic effects, both at 27 and $60 \mathrm{MHz}$, especially in absolute values. Indeed, when the Maxwell solver is included, the electron density becomes higher at both 27 and $60 \mathrm{MHz}$, which yields higher electron impact reaction rates, and gives rise to a higher plasma density and higher fluxes above the wafer. When the frequency is low, i.e., $27 \mathrm{MHz}$, no obvious electromagnetic effects on the shapes of the $\mathrm{CF}_{3}^{+}$and $\mathrm{F}^{-}$densities and on the fluxes of the neutral species and ions are detected. However, as the frequency increases to $60 \mathrm{MHz}$, the differences between the plasma characteristics obtained with and without Maxwell solver become more obvious. A nonuniform $\mathrm{F}^{-}$ density distribution is observed, and the $\mathrm{O}$ atom flux above the bottom electrode is characterized by a pronounced maximum at the edge due to the profound influence of the electromagnetic effects at $60 \mathrm{MHz}$. Finally, the electromagnetic effects act differently on the electron density profile at 27 and $60 \mathrm{MHz}$, because the profile is more uniform in the case with the electromagnetic effects at $27 \mathrm{MHz}$, whereas it becomes less uniform at $60 \mathrm{MHz}$. This can be explained by the fact that the radial inductive electric field, which is characterized by a prominent off-axis peak, plays a dominant role in the plasma distribution at $60 \mathrm{MHz}$.

\section{Effect of adding a low frequency power on the plasma uniformity}

As shown in Sec. IV B, the plasma density becomes more nonuniform along the radial direction at higher frequency. In plasma processing applications, it is important to improve the plasma uniformity, as it affects the uniformity of the etch and deposition processes. Therefore, we have applied an additional LF power of $2 \mathrm{MHz}$ on the bottom electrode, varying from 0 to $1000 \mathrm{~W}$, to elucidate whether this can 

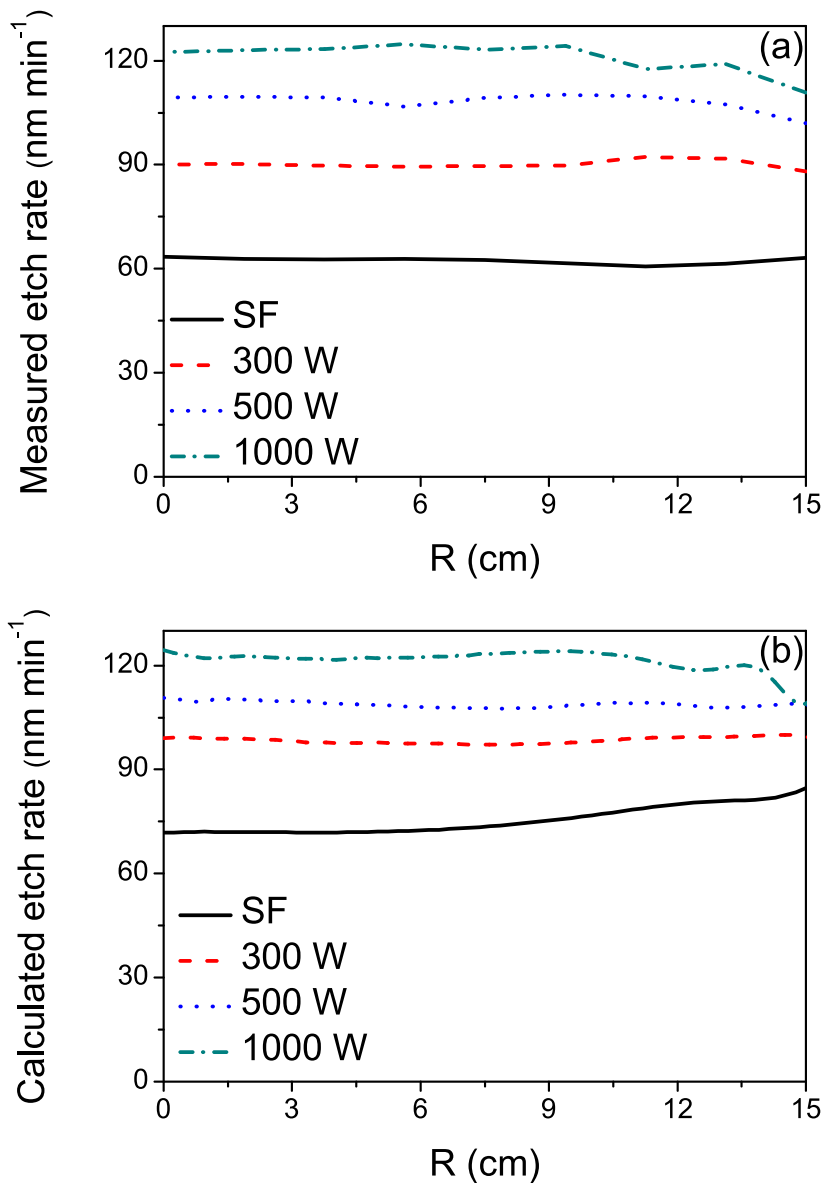

FIG. 9. (Color online) Radial distributions of the measured (a) and calculated (b) etch rates in a $\mathrm{SF} \mathrm{CF}_{4} / \mathrm{O}_{2}$ discharge at $27 \mathrm{MHz}$, and a DF discharge sustained at $27+2 \mathrm{MHz}$, and different LF powers. The HF power is fixed at $300 \mathrm{~W}$. The other conditions are also the same as in Fig. 3.

improve the plasma uniformity. The HF is fixed at 27 and 60 $\mathrm{MHz}$, with a power of $300 \mathrm{~W}$.

In this section, we will show the measured etch rates across the wafer, and compare them with the calculated fluxes of various species to the wafer, as obtained by the model, at two different HFs (27 and $60 \mathrm{MHz}$ ) and various values of the LF power. We defined a so-called nonuniformity degree $\alpha$ as follows:

$$
\alpha=\left(R_{\max }-R_{\min }\right) / 2 R_{\text {ave }},
$$

where $R_{\max }, R_{\min }$, and $R_{\text {ave }}$ are the maximum, the minimum, and the average values of the etch rate.

The evolution of the measured etch rate as a function of LF power in the discharge with a HF source of $27 \mathrm{MHz}$ at $300 \mathrm{~W}$ is presented in Fig. 9(a). When the LF source is switched off, the distribution of the etch rate is uniform along the radial direction $(\alpha \approx 2.2 \%)$, and the absolute value is very low, i.e., $62 \mathrm{~nm} \min ^{-1}$. By switching on the LF source, the etch rate increases drastically, and it becomes significantly higher at higher LF power values. This indicates that the LF power plays an important role in producing a higher density plasma. At a LF power of $300 \mathrm{~W}$, the averaged etch rate is in the order of $90 \mathrm{~nm} \mathrm{~min}{ }^{-1}$, and the uniformity is comparable $(\alpha \approx 2.3 \%)$. As the LF power increases further to 500 and
$1000 \mathrm{~W}$, the averaged etch rate increases further, to about $121 \mathrm{~nm} \mathrm{~min} \mathrm{~m}^{-1}$ at $1000 \mathrm{~W}$, but the uniformity becomes worse. Indeed, the etch rate reaches a minimum near the radial edge of the bottom electrode, and the nonuniformity degree at 1000 $\mathrm{W}(\alpha \approx 5.8 \%)$ is two to three times higher than in the case without LF source.

In the etch process, the material can be removed from the surface by sputtering, pure chemical etching, ion energy driven etching, and ion inhibitor etching. It is known that the $\mathrm{F}$ atoms can react with silicon and silicon dioxide, forming volatile compounds without ion bombardment, which means that the $\mathrm{F}$ atoms play a role in determining the etch rate. ${ }^{1}$ However, for a given $\mathrm{F}$ atom flux, the etch process can be enhanced, and the etch rate can increase by a factor of 5-10 due to the bombardment of energetic ions. ${ }^{1}$ Hence, the etch rate is also influenced by the ion flux above the wafer. Moreover, the discharge does not only generate the etchants and energetic ions, but also the precursors for deposition of polymer films, such as $\mathrm{CF}_{\mathrm{x}}$-based layers. It is known that $\mathrm{CF}_{3}$ radicals do not contribute much to the polymer deposition, due to their low sticking probability. ${ }^{66}$ Hence, the $\mathrm{CF}_{2}$ radicals are considered as the main species for polymer deposition, which will reduce the etch rate.

In order to quantitatively calculate the etch rates with the model, a full surface chemistry module with a large number of reactions needs to be built, as described in Ref. 45, for which more input data than just the sticking coefficients are needed, which are not commonly available. Therefore, in this work, a simple formula is employed to estimate the etch rates under different conditions, and to compare them in a qualitative way with experimental data. The etch rate in pure $\mathrm{CF}_{4}$ discharges can be estimated from

$$
E_{\mathrm{Si}}=\frac{\Gamma_{\mathrm{F}}-(4-x) \Gamma_{\mathrm{CF}_{\mathrm{x}}}}{2 n_{\mathrm{Si}}},
$$

where $\Gamma_{\mathrm{F}}$ and $\Gamma_{\mathrm{CF}_{\mathrm{x}}}$ are the net fluxes of $\mathrm{F}$ and $\mathrm{CF}_{\mathrm{x}}$ toward the wafer, respectively. ${ }^{1}$ According to this formula, it is clear that the etch rate increases with the F flux, and decreases with the $\mathrm{CF}_{\mathrm{x}}$ fluxes, as they cause deposition of polymer films which cannot be removed from the surface in the absence of ion bombardment, and this accordingly reduces the etch rate. In addition, when $\mathrm{O}_{2}$ is added to $\mathrm{CF}_{4}$, the $\mathrm{O}$ atoms will oxidize the surface, which will accordingly reduce the etch rate as well. Under this condition, the etch rate is given by

$$
E_{\mathrm{Si}}=\frac{\Gamma_{\mathrm{F}}}{n_{\mathrm{Si}}\left(1+\eta_{\mathrm{O}} \Gamma_{\mathrm{O}} / \Gamma_{\mathrm{F}}\right)},
$$

where $\eta_{\mathrm{O}}$ gives the competitive efficiency for $\mathrm{O}$ atoms over $\mathrm{F}$ atoms to be adsorbed. ${ }^{1}$ For a given $\eta_{\mathrm{O}}$, the etch rate becomes lower as the $\mathrm{O}$ atom flux increases.

Based on the equations mentioned above, the etch rate in our case $\left(\mathrm{CF}_{4} / \mathrm{O}_{2}\right.$ plasma) can be approximately expressed as

$$
E_{\mathrm{Si}}=\text { factor }_{1} \frac{\Gamma_{\mathrm{F}}-f\left(\Gamma_{\mathrm{CF}_{\mathrm{x}}}\right)}{1+\text { factor }_{2} \Gamma_{\mathrm{O}} / \Gamma_{\mathrm{F}}},
$$

where $f\left(\Gamma_{\mathrm{CF}_{\mathrm{x}}}\right)$ is a function of the $\mathrm{CF}_{2}$ radical flux. Indeed, as mentioned above, $\mathrm{CF}_{2}$ is the most important species for 

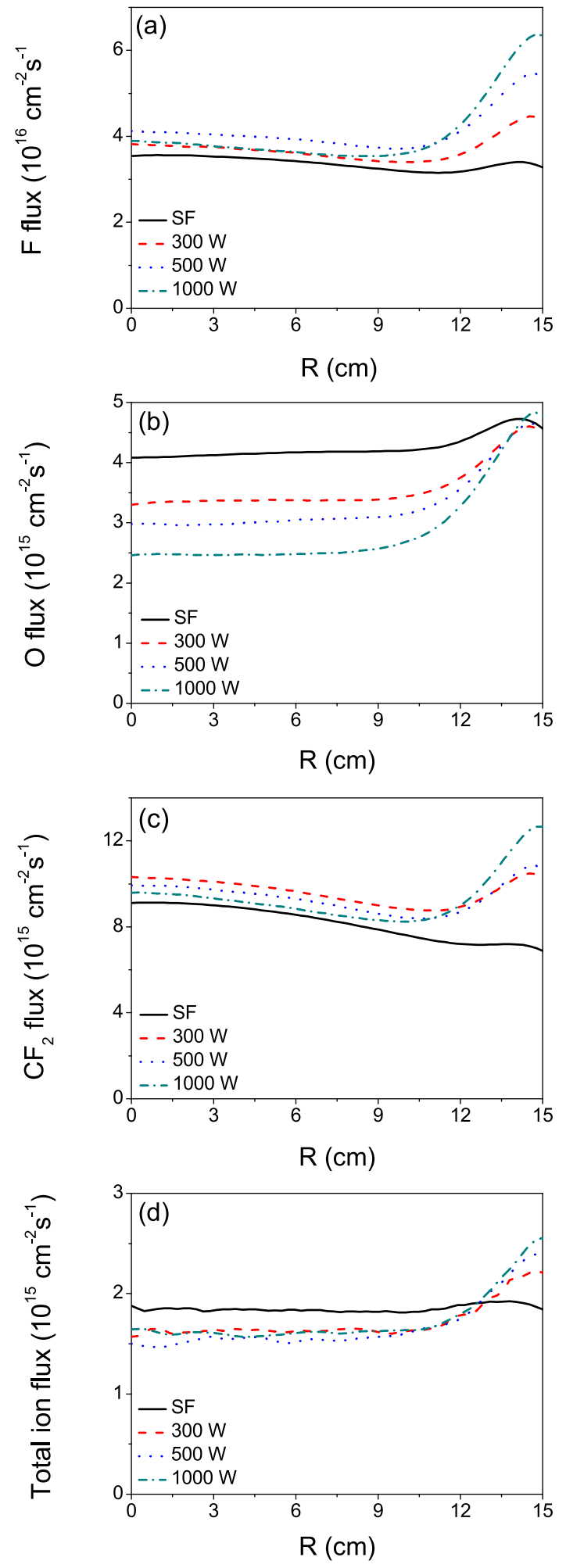

FIG. 10. (Color online) Radial distributions of the $\mathrm{F}$ atom flux (a), O atom flux (b), $\mathrm{CF}_{2}$ radical flux (c), and total positive ion flux (d) at the bottom electrode in a SF $\mathrm{CF}_{4} / \mathrm{O}_{2}$ discharge at $27 \mathrm{MHz}$, and a DF discharge sustained at $27+2 \mathrm{MHz}$, and different LF powers. The HF power is fixed at $300 \mathrm{~W}$. The other conditions are also the same as in Fig. 3.

polymer deposition. By fitting this function and the values of factor $_{1}$ and factor 2 according to the experimental data in one case at the $\mathrm{HF}$ of 27 and $60 \mathrm{MHz}$, the etch rate is estimated for all the other cases. The radial distributions of the etch
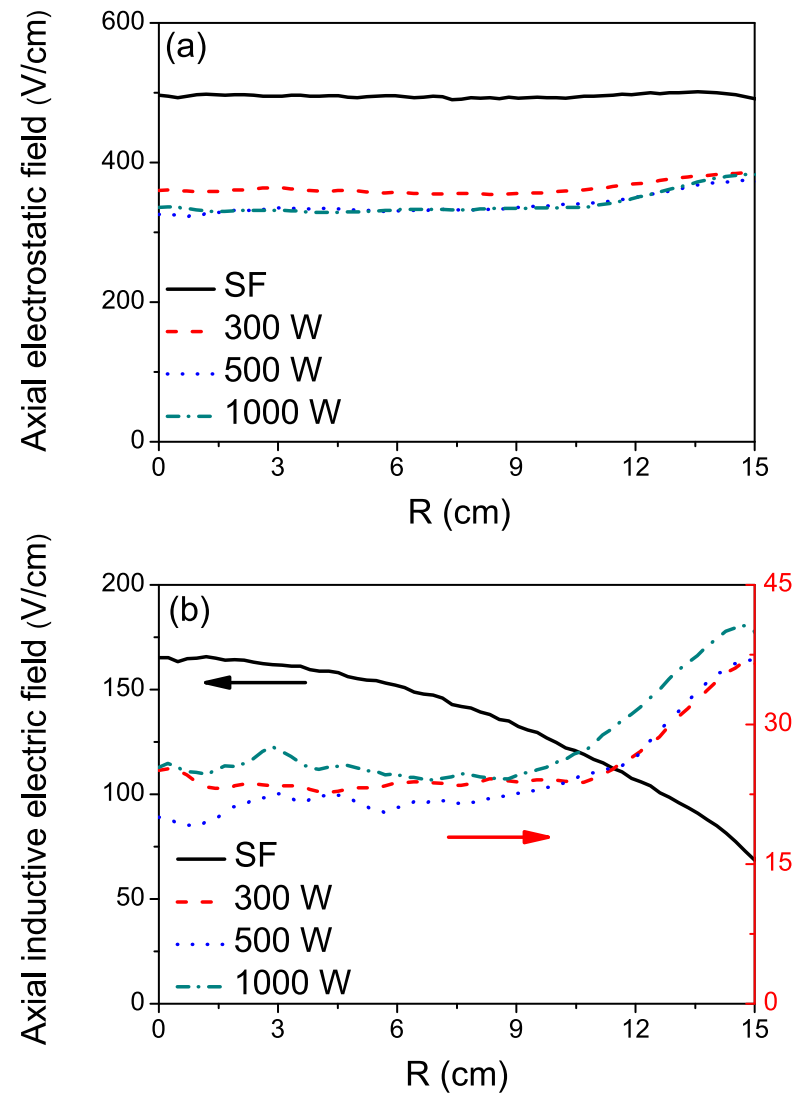

FIG. 11. (Color online) Radial distributions of the axial electrostatic field (a) and axial inductive electric field (b) at the bottom electrode in a $\mathrm{SF} \mathrm{CF}_{4} / \mathrm{O}_{2}$ discharge at $27 \mathrm{MHz}$, and a DF discharge sustained at $27+2 \mathrm{MHz}$, and different LF powers. The HF power is fixed at $300 \mathrm{~W}$. The other conditions are also the same as in Fig. 3.

rates calculated in this way at $27 \mathrm{MHz}$ are shown in Fig. 9(b). By comparing with the experimental data, it is clear that the calculated etch rate in the SF discharge is less uniform, being higher at the edge. When the LF source is added with a power of $300 \mathrm{~W}$, the calculated etch rate increases, and it becomes more uniform along the radial direction, as was also observed in the experiment [see Fig. 9(a)]. As the LF power increases to $500 \mathrm{~W}$, the calculated etch rate increases from 99 to $110 \mathrm{~nm} \mathrm{~min}^{-1}$, and the radial uniformity is still good. However, the uniformity becomes worse when the LF power increases further to $1000 \mathrm{~W}$, which is similar to the experimental results. Although this simple formula can of course not describe the entire surface chemistry in an accurate way, it can help us to understand the evolution of the etch rates under different conditions.

In addition, it should be noted that in the etch process, the etchants diffuse or flow to the surface, where they are adsorbed and react to form the product. The etched species are desorbed from the surface, diffuse or flow into the gas phase and react with the species in the discharge. Besides, the etched species in the gas phase can also be adsorbed back onto the surface again. Therefore, the concentration of the etch products in the plasma depends on the etch rate and the gas flow (residence time). We calculated before that in an inductively coupled plasma discharge at 40 mTorr with an etch rate of $300 \mathrm{~nm} / \mathrm{min}$ at $100 \mathrm{sccm}$ gas flow, the total 

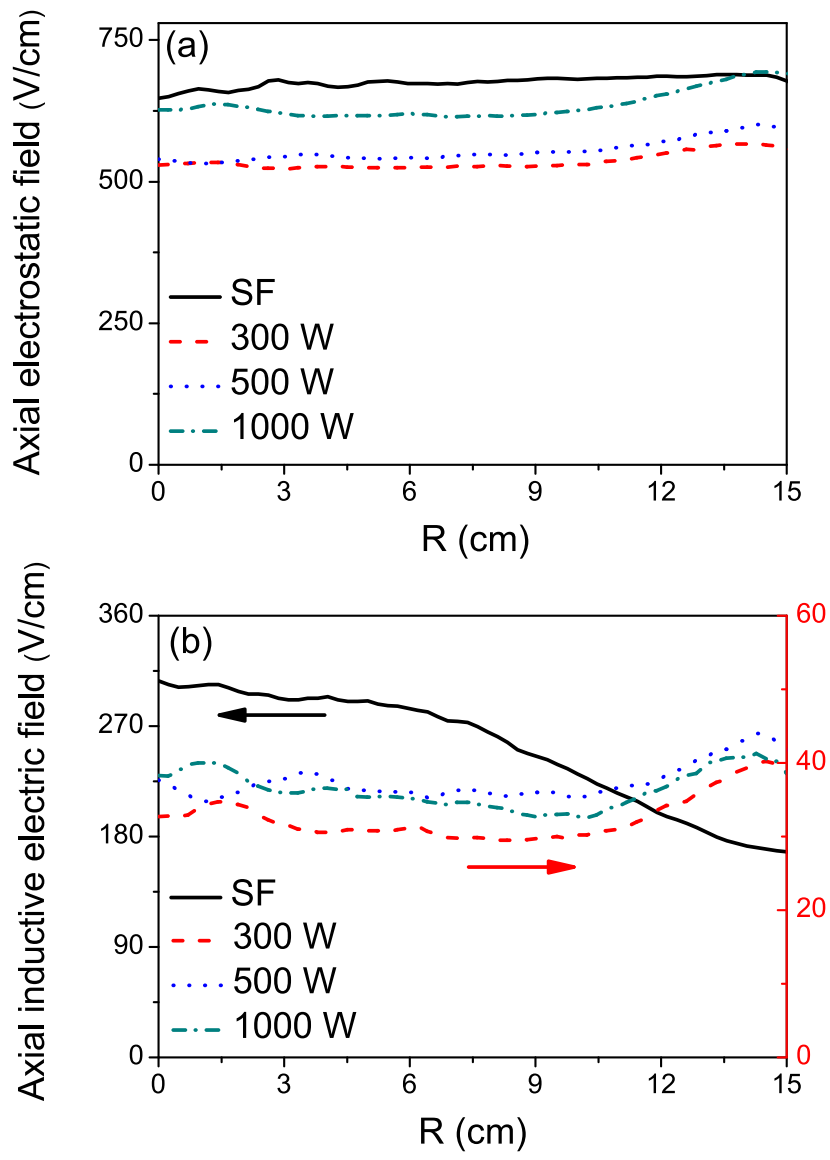

FIG. 12. (Color online) Radial distributions of the axial electrostatic field (a) and axial inductive electric field (b) at the bottom electrode in a $\mathrm{SF} \mathrm{CF}_{4} / \mathrm{O}_{2}$ discharge at $60 \mathrm{MHz}$, and a DF discharge sustained at $60+2 \mathrm{MHz}$, and different LF powers. The HF power is fixed at $300 \mathrm{~W}$. The other conditions are also the same as in Fig. 3.

fraction of the etch products in the plasma is about $15 \% .^{46}$ Since the pressure is fixed at $50 \mathrm{mTorr}$ in this work, and the maximum of the etch rate is three times lower $(121 \mathrm{~nm} / \mathrm{min})$ than in Ref. 46, we expect that the fraction of the etch products in the plasma is about $5 \%$ in this case. Therefore, we believe that the etch products have no significant influence on the discharge.

Figure 10 shows the radial distributions of the fluxes of the $\mathrm{F}$ atoms, the $\mathrm{O}$ atoms, the $\mathrm{CF}_{2}$ radicals and the total positive ions toward the wafer, calculated for the same conditions as in Fig. 9, as these four fluxes will determine the etch rate. From Figs. 10(a) and 10(b), it can be deduced that when there is no LF source, the lower F atom flux and higher $\mathrm{O}$ atom flux lead to a lower etch rate in the SF discharge, as illustrated in Fig. 9. When the LF source is switched on with a power of $300 \mathrm{~W}$, the $\mathrm{F}$ atom flux increases slightly, whereas the $\mathrm{O}$ atom flux exhibits a considerable drop, thus the etch process is enhanced, explaining the higher etch rate observed in Fig. 9. The significant drop of the $\mathrm{O}$ atom flux can be explained by the weaker axial electrostatic field above the bottom electrode in a DF discharge (as will be discussed below), which reduces the electron impact dissociation of $\mathrm{O}_{2}$ and yields a lower $\mathrm{O}$ atom flux. Although the $\mathrm{F}$ atom flux and the total positive ion flux are characterized by
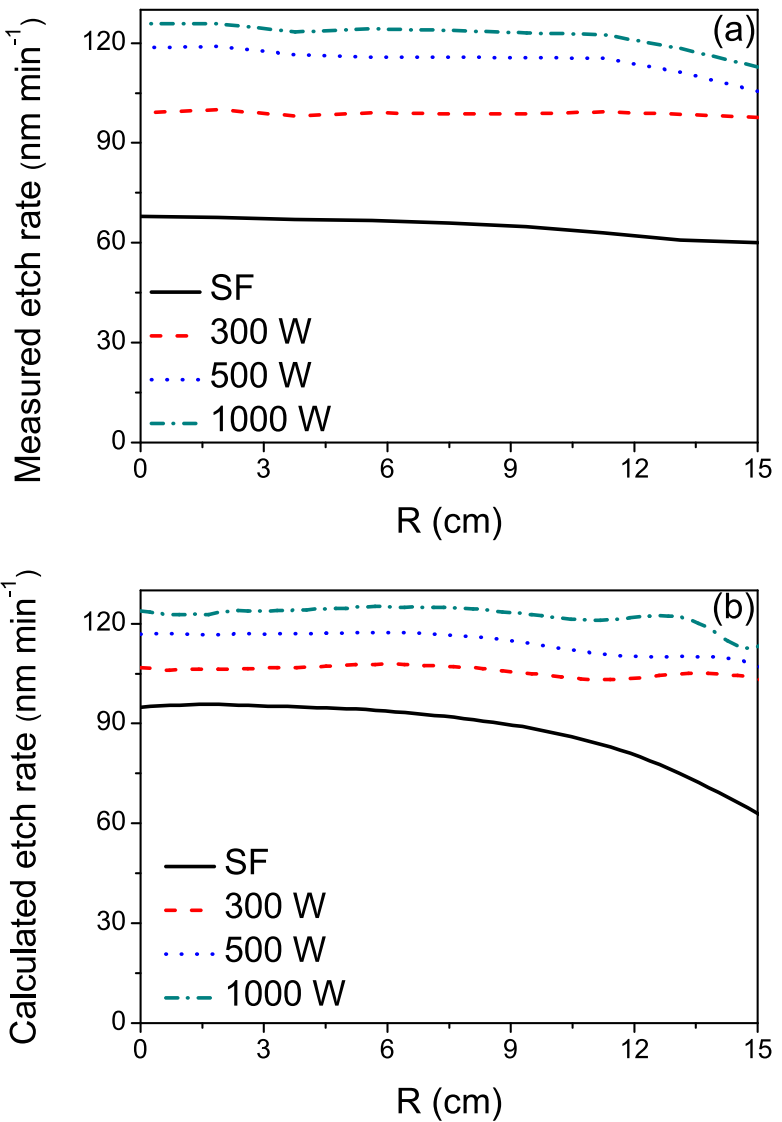

FIG. 13. (Color online) Radial distributions of the measured (a) and calculated (b) etch rates in a SF $\mathrm{CF}_{4} / \mathrm{O}_{2}$ discharge at $60 \mathrm{MHz}$, and a $\mathrm{DF}$ discharge sustained at $60+2 \mathrm{MHz}$, and different LF powers. The HF power is fixed at $300 \mathrm{~W}$. The other conditions are also the same as in Fig. 3.

a clear off-axis peak, the higher $\mathrm{O}$ atom and $\mathrm{CF}_{2}$ radical fluxes near the radial edge of the wafer reduce the etch rate there, and this explains the rather uniform etch rate at this condition, as was shown in Fig. 9. As the LF power increases to 500 and $1000 \mathrm{~W}$, the $\mathrm{F}$ atom and total ion fluxes become more nonuniform, but the same applies for the $\mathrm{O}$ atom flux and the $\mathrm{CF}_{2}$ radical flux, which are also characterized by strong off-axis peaks, caused by the prominent power deposition near the radial edge due to the skin effect. It is clear that these peaks have now a dominant influence on the etch rate, because the overall etch rate is at minimum near the radial edge at a LF power of $1000 \mathrm{~W}$.

Figure 11 illustrates the radial distributions of the axial electrostatic (a) and inductive electric (b) fields at various LF powers. The lower axial electrostatic field in DF discharges, as mentioned above, is indeed obvious from Fig. 11(a). Furthermore, it is clear that the axial inductive electric field exhibits a broad maximum in the center in the SF discharge, whereas it shifts to an edge-high profile with much lower absolute values when the LF source is switched on. This indicates that the electromagnetic effects become weaker by adding a LF power.

As the $\mathrm{HF}$ increases to $60 \mathrm{MHz}$, the electromagnetic effects play a major role in the SF discharge, as we have illustrated in Sec. IV B, and therefore, adding the LF power has a different effect on the plasma uniformity. In a SF 

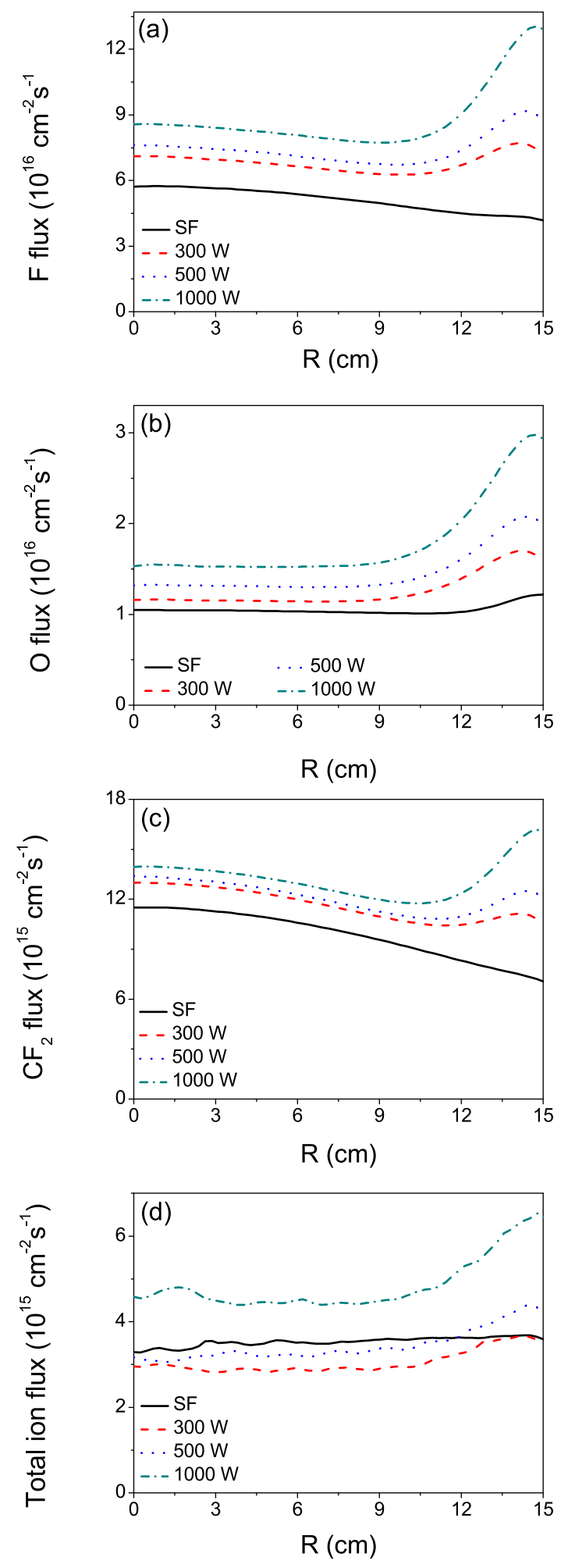

FIG. 14. (Color online) Radial distributions of the $\mathrm{F}$ atom flux (a), $\mathrm{O}$ atom flux (b), $\mathrm{CF}_{2}$ radical flux (c), and total positive ion flux (d) at the bottom electrode in a $\mathrm{SF} \mathrm{CF}_{4} / \mathrm{O}_{2}$ discharge at $60 \mathrm{MHz}$, and a DF discharge sustained at $60+2 \mathrm{MHz}$, and different LF powers. The HF power is fixed at $300 \mathrm{~W}$. The other conditions are also the same as in Fig. 3.

discharge sustained at $60 \mathrm{MHz}$ and $300 \mathrm{~W}$, the axial electrostatic field is rather uniform [see Fig. 12(a)], but the axial inductive electric field is characterized by a maximum at the center [i.e., $307 \mathrm{~V} / \mathrm{cm}$, cf. Fig. 11(b)], which demonstrates again the strong electromagnetic effects under this condition. Therefore, the etch rate is slightly higher at the reactor center, and the nonuniformity degree is about $6.1 \%$ [see Fig. 13(a)]. When the LF source is added with a power of $300 \mathrm{~W}$, the electromagnetic effects are clearly suppressed, as is demonstrated in Fig. 12(b). Indeed, a more uniform axial inductive electric field distribution is observed with only a slightly higher value near the edge, and the magnitude is almost one order lower than in the case without LF source [i.e., maximum of about $42 \mathrm{~V} / \mathrm{cm}$, cf. Fig. 12(b)]. Therefore, the uniformity of the etch rate becomes much better [see Fig. 13(a)], with a value of $\alpha$ equal to $0.7 \%$, which is about one order lower than the value in the SF discharge. Moreover, the amplitude of the etch rate increases by about $50 \%$ (i.e., around $65 \mathrm{~nm} \mathrm{~min}^{-1}$ in the SF discharge, and around $99 \mathrm{~nm}$ $\min ^{-1}$ at a LF power of $300 \mathrm{~W}$ ). This demonstrates that the radial uniformity can be improved by adding a LF source. However, when a LF power of 500 or $1000 \mathrm{~W}$ is added, the etch rate at the reactor center increases significantly, whereas the etch rate near the radial edge increases only slightly, and therefore, the uniformity becomes worse again (i.e., with a maximum etch rate at the reactor center, and $\alpha \approx 5.8 \%$ at $500 \mathrm{~W}$ and $\alpha \approx 5.3 \%$ at $1000 \mathrm{~W}$ ).

The calculated etch rate profiles at the $\mathrm{HF}$ of $60 \mathrm{MHz}$ with different LF powers are shown in Fig. 13(b). The etch rate is characterized by a center high profile when the LF source is switched off, due to the significant electromagnetic effects. By switching on the LF source, the etch rate at the radial edge increases faster than at the center, which produces a more uniform etch rate at a LF power of $300 \mathrm{~W}$. However, as the LF power increases further to 500 and 1000 $\mathrm{W}$, the etch rate at the reactor center is slightly higher than at the radial edge, and the uniformity becomes worse again. Although the absolute values of the calculated etch rates are somewhat different from those measured in the experiment, the trend with rising LF power is the same.

In order to understand these different etch rate profiles, the fluxes of $\mathrm{F}, \mathrm{O}$, and $\mathrm{CF}_{2}$ and the total positive ion flux are presented in Fig. 14, for the same conditions as in Figs. 12 and 13. When there is no LF power, the F atom flux at the center is somewhat higher than near the edge [Fig. 14(a)], whereas the total positive ion flux exhibits a uniform distribution along the radial direction [Fig. 14(d)]. The O atom flux is somewhat higher near the edge, whereas the $\mathrm{CF}_{2}$ flux is characterized by a broad maximum in the center. The combination of these profiles leads to an etch rate which is slightly higher at the reactor center. By switching on the LF source at $300 \mathrm{~W}$, the $\mathrm{F}$ atom flux near the radial edge shows a pronounced increase, which enhances the etch process there. However, the $\mathrm{O}$ atom flux reduces the etch rate at the radial edge, yielding a good uniformity of the etch rate, as is clear from Fig. 13. When the LF power increases further to 500 and $1000 \mathrm{~W}$, although the off-axis peaked profiles of the $\mathrm{F}$ atom flux and the total positive ion flux become more significant, the prominent peaks of the $\mathrm{CF}_{2}$ and $\mathrm{O}$ atom fluxes near the radial edge have a dominant influence on the etch process, resulting in a decrease of the overall etch rate near the edge. As a consequence, the etch rate profile shows a 
maximum near the center, and the uniformity becomes worse again, as was indeed clear from Fig. 13.

\section{CONCLUSIONS}

In this work, a two-dimensional hybrid fluid-Monte Carlo model combined with a full-wave Maxwell solver, i.e., the so-called HPEM, is employed to investigate the plasma behavior in a SF and DF CCP operating in $\mathrm{CF}_{4} / \mathrm{O}_{2}$. After showing the typical plasma composition, we demonstrate by examining the spatial distributions of the electron density, $\mathrm{CF}_{3}^{+}$density, $\mathrm{F}^{-}$density, and of the fluxes of various species toward the wafer, that the electromagnetic effects have an important influence on the plasma properties, and the plasma uniformity, of a SF CCP, both at 27 and $60 \mathrm{MHz}$. However, it does not mean that the electromagnetic effects are always dominant. When these effects are not pronounced, or when the focus of the research would be on other aspects in the discharge, the electrostatic approximation also works well.

Therefore, to find out whether adding a LF power can improve the plasma (and etch rate) uniformity, we have performed a detailed study on the plasma uniformity in both SF and DF CCPs, at a HF of 27 and $60 \mathrm{MHz}$ and a LF of 2 $\mathrm{MHz}$, for different values of LF power. For this purpose, we have measured the etch rate in this range of conditions. At a $\mathrm{HF}$ of $27 \mathrm{MHz}$, the etch rate is rather uniform in a SF discharge, as well as in a DF discharge when adding a LF power of $300 \mathrm{~W}$; the latter enhances the etch rate but does not affect the uniformity. However, adding a higher LF power (of 500 or $1000 \mathrm{~W}$ ) increases the etch rate further, but the uniformity becomes worse. On the other hand, when the discharge is sustained at a $\mathrm{HF}$ of $60 \mathrm{MHz}$ and $300 \mathrm{~W}$, the etch rate is less uniform, with slightly higher values at the reactor center in the SF discharge, and the uniformity becomes much better when a LF power of $300 \mathrm{~W}$ is added. As the LF power increases further to 500 and $1000 \mathrm{~W}$, the uniformity becomes worse again, with a minimum near the radial edge. This behavior of the etch rate under various conditions could be explained by examining the fluxes of various species above the wafer, playing a role in the etch process, i.e., the $\mathrm{F}$ atoms, the $\mathrm{O}$ atoms, the $\mathrm{CF}_{2}$ radicals and the ions, as well as from the radial profiles of the axial electrostatic and inductive electric fields. From these studies, we can conclude that the electromagnetic effects have an important influence on the plasma behavior, especially on the plasma uniformity in $\mathrm{CF}_{4} / \mathrm{O}_{2}$ discharges sustained by $\mathrm{HF}$ and VHF sources. By adding a LF source with a moderate power (e.g., $300 \mathrm{~W}$ ), the nonuniformity caused by the electromagnetic effects, especially in the $60 \mathrm{MHz}$ case, can be suppressed. However, when the LF power is too high (e.g., 500 or $1000 \mathrm{~W}$ ), the uniformity becomes worse again. This is very important to realize, as it can help us to optimize the plasma processing.

\section{ACKNOWLEDGMENTS}

This work was supported by the National Natural Science Foundation of China (Grant Nos. 11405019 and 11335004), the Important National Science \& Technology Specific Project (Grant No 2011ZX02403-001), the China
Postdoctoral Science Foundation (Grant No. 2014M561219), the Fundamental Research Funds for Central Universities (Grant No. DUT14RC(3)071), the International Science \& Technology Cooperation Program of China (Grant No 2012DFG02150), the joint research project in the framework of the agreement between MOST and FWO, and the University of Antwerp BOF fellowship for Y.-R. Zhang. The authors are very grateful to M. J. Kushner for providing the HPEM code. This work was carried out in part using the Turing HPC infrastructure at the CalcUA core facility of the Universiteit Antwerpen, a division of the Flemish Supercomputer Center VSC, funded by the Hercules Foundation, the Flemish Government (department EWI) and the University of Antwerp.

${ }^{1}$ M. A. Lieberman and A. J. Lichtenberg, Principles of Plasma Discharges and Materials Processing, 2nd ed. (Wiley, New York, 2005).

${ }^{2}$ M. Haverlag, A. Kono, D. Passchier, G. M. W. Kroesen, W. J. Goedheer, and F. J. de Hoog, J. Appl. Phys. 70, 3472 (1991).

${ }^{3}$ W. Schwarzenbach, G. Cunge, and J. P. Booth, J. Appl. Phys. 85, 7562 (1999).

${ }^{4}$ M. Haverlag, W. W. Stoffels, E. Stoffels, G. M. W. Kroesen, and F. J. de Hoog, J. Vac. Sci. Technol., A 14, 384 (1996).

${ }^{5}$ J. P. Booth, G. Cunge, P. Chabert, and N. Sadeghi, J. Appl. Phys. 85, 3097 (1999).

${ }^{6}$ P. Fendel, A. Francis, and U. Czarnetzki, Plasma Sources Sci. Technol. 14, 1 (2005).

${ }^{7}$ E. Gogolides, M. Stathakopoulos, and A. Boudouvis, J. Phys. D: Appl. Phys. 27, 1878 (1994).

${ }^{8}$ N. V. Mabtzaris, A. Boudouvis, and E. Gogolides, J. Appl. Phys. 77, 6169 (1995).

${ }^{9}$ S. Segawa, M. Kurihara, N. Nakano, and T. Makabe, Jpn. J. Appl. Phys., Part 1 38, 4416 (1999).

${ }^{10}$ S. Y. So, A. Oda, H. Sugawara, and Y. Sakai, J. Phys. D: Appl. Phys. 34, 1919 (2001).

${ }^{11}$ K. Denpoh and K. Nanbu, J. Vac. Sci. Technol., A 16, 1201 (1998).

${ }^{12}$ V. Georgieva, A. Bogaerts, and R. Gijbels, J. Appl. Phys. 93, 2369 (2003).

${ }^{13}$ O. V. Proshina, T. V. Rakhimova, A. T. Rakhimov, and D. G. Voloshin, Plasma Sources Sci. Technol. 19, 065013 (2010).

${ }^{14}$ C. J. Mogab, A. C. Adams, and D. L. Flamm, J. Appl. Phys. 49, 3796 (1978).

${ }^{15}$ R. d'Agostino, F. Cramarossa, S. De Benedistis, and G. Ferraro, J. Appl. Phys. 52, 1259 (1981).

${ }^{16}$ V. M. Donnelly, D. L. Flamm, W. C. Dautremont-Smith, and D. J. Werder, J. Appl. Phys. 55, 242 (1984).

${ }^{17}$ J. W. Kim, Y. C. Kim, and W. J. Lee, J. Appl. Phys. 78, 2045 (1995).

${ }^{18}$ I. C. Plumb and K. R. Ryan, Plasma Chem. Plasma Process. 6, 205 (1986).

${ }^{19}$ M. Dalvie and K. F. Jensen, J. Vac. Sci. Technol., A 8, 1648 (1990).

${ }^{20}$ R. Knizikevicius, A. Galdikas, and A. Grigonis, Vacuum 66, 39 (2002).

${ }^{21}$ T. Kimura and M. Noto, J. Appl. Phys. 100, 063303 (2006).

${ }^{22}$ H. A. Goto, H. D. Lowe, and T. Ohmi, J. Vac. Sci. Technol., A 10, 3048 (1992).

${ }^{23}$ J. P. Booth, C. S. Corr, G. A. Curley, J. Jolly, J. Guillon, and T. Foldes, Appl. Phys. Lett. 88, 151502 (2006).

${ }^{24}$ J. Schulze, A. Derzsi, and Z. Donko, Plasma Sources Sci. Technol. 20, 045008 (2011).

${ }^{25}$ S. H. Song and M. J. Kushner, Plasma Sources Sci. Technol. 21, 055028 (2012).

${ }^{26}$ M. A. Lieberman, J. P. Booth, P. Chabert, J. M. Rax, and M. M. Turner, Plasma Sources Sci. Technol. 11, 283 (2002).

${ }^{27}$ Y. R. Zhang, X. Xu, S. X. Zhao, A. Bogaerts, and Y. N. Wang, Phys. Plasmas 17, 113512 (2010).

${ }^{28}$ G. A. Hebner, E. V. Barnat, P. A. Miller, A. M. Paterson, and J. P. Holland, Plasma Sources Sci. Technol. 15, 879 (2006).

${ }^{29}$ E. V. Barnat, P. A. Miller, G. A. Hebner, A. M. Paterson, T. Panagopoulos, E. Hammond, and J. Holland, Appl. Phys. Lett. 90, 201503 (2007).

${ }^{30}$ K. Bera, S. Rauf, K. Ramaswamy, and K. Collins, J. Appl. Phys. 106, 033301 (2009). 
${ }^{31}$ Y. Yang and M. J. Kushner, Plasma Sources Sci. Technol. 19, 055011 (2010).

${ }^{32}$ Y. Yang and M. J. Kushner, Plasma Sources Sci. Technol. 19, 055012 (2010).

${ }^{33}$ L. Sansonnens and J. Schmitt, Appl. Phys. Lett. 82, 182 (2003).

${ }^{34}$ H. Schmidt, L. Sansonnens, A. A. Howling, Ch. Hollenstein, M. Elyaakoubi, and J. P. M. Schmitt, J. Appl. Phys. 95, 4559 (2004).

${ }^{35}$ P. Chabert, J. L. Raimbault, J. M. Ram, and A. Perret, Phys. Plasmas 11, 4081 (2004).

${ }^{36}$ Y. Takeuchi, Y. Nawata, K. Ogawa, A. Serizawa, Y. Yamauchi, and M. Murata, Thin Solid Films 386, 133 (2001).

${ }^{37}$ H. Takatsuka, M. Noda, Y. Yonekura, Y. Takeuchi, and Y. Yamauchi, Sol. Energy 77, 951 (2004).

${ }^{38}$ Y. Yang and M. J. Kushner, J. Phys. D: Appl. Phys. 43, 152001 (2010).

${ }^{39}$ D. Sung, S. Jeong, Y. Park, V. N. Volynets, A. G. Ushakov, and G.-H. Kim, J. Vac. Sci. Technol., A 27, 13 (2009).

${ }^{40}$ K. Bera, S. Rauf, and K. Collins, IEEE Trans. Plasma Sci. 36, 1366 (2008).

${ }^{41}$ Y. R. Zhang, X. Xu, A. Bogaerts, and Y. N. Wang, J. Phys. D: Appl. Phys. 45, 015202 (2012).

${ }^{42}$ Y. R. Zhang, A. Bogaerts, and Y. N. Wang, J. Phys. D: Appl. Phys. 45, 485204 (2012).

${ }^{43}$ P. Chabert and N. Braithwaite, Physics of Radio-Frequency Plasmas (Cambridge, New York, 2011).

${ }^{44}$ M. J. Kushner, J. Phys. D: Appl. Phys. 42, 194013 (2009).

${ }^{45}$ S. Tinck, W. Boullart, and A. Bogaerts, J. Phys. D: Appl. Phys. 42, 095204 (2009).

${ }^{46}$ S. Tinck, W. Boullart, and A. Bogaerts, Plasma Sources Sci. Technol. 20, 045012 (2011).

${ }^{47}$ S. Tinck and A. Bogaerts, Plasma Process. Polym. 9, 522 (2012).

${ }^{48}$ See: http://www.lamresearch.com/.

${ }^{49}$ R. A. Bonham, Jpn. J. Appl. Phys., Part 1 33, 4157 (1994).
${ }^{50}$ E. Meeks, R. S. Larson, S. R. Vosen, and J. W. Shon, J. Electrochem. Soc. 144, 357 (1997).

${ }^{51}$ M. Hayashi and T. Nimura, J. Appl. Phys. 54, 4879 (1983).

${ }^{52}$ S. X. Zhao, F. Gao, Y. N. Wang, and A. Bogaerts, Plasma Sources Sci. Technol. 21, 025008 (2012).

${ }^{53}$ A. V. Phelps, JILA Information Center Report No. 28, University of Colorado (1985).

${ }^{54}$ A. V. Vasenkov, X. Li, G. S. Oehrlein, and M. J. Kushner, J. Vac. Sci. Technol., A 22, 511 (2004).

${ }^{55}$ R. R. Laher and F. R. Gilmore, J. Phys. Chem. Ref. Data 19, 277 (1990).

${ }^{56}$ W. Liu and G. A. Victor, Astrophys. J. 435, 909 (1994).

${ }^{57}$ J. J. Lowke, A. V. Phelps, and B. W. Irwin, J. Appl. Phys. 44, 4664 (1973).

${ }^{58}$ D. Eremin, T. Hemke, R. P. Brinkmann, and T. Mussenbrock, J. Phys. D: Appl. Phys. 46, 084017 (2013).

${ }^{59}$ S. Tinck, E. C. Neyts, and A. Bogaerts, J. Phys. Chem. C 118, 30315 (2014).

${ }^{60}$ V. A. Godyak and A. S. Khanneh, IEEE Trans. Plasma Sci. PS-14, 112 (1986).

${ }^{61} \mathrm{Ph}$. Belenguer and J. P. Boeuf, Phys. Rev. A 41, 4447 (1990).

${ }^{62}$ X. P. Xu, S. Rauf, and M. J. Kushner, J. Vac. Sci. Technol., A 18, 213 (2000).

${ }^{63}$ L. G. Christophorou, J. K. Olthoff, and M. V. V. S. Rao, J. Phys. Chem. Ref. Data 25, 1341 (1996).

${ }^{64}$ T. Mussenbrock, T. Hemke, D. Ziegler, R. P. Brinkmann, and M. Klick, Plasma Sources Sci. Technol. 17, 025018 (2008).

${ }^{65}$ H. Hayashi, S. Morishita, T. Tatsumi, Y. Hikosaka, S. Noda, H. Nakagawa, S. Kobayashi, M. Inoue, and T. Hoshino, J. Vac. Sci. Technol., A 17, 2557 (1999).

${ }^{66}$ S. X. Zhao, F. Gao, Y. N. Wang, and A. Bogaerts, Plasma Sources Sci. Technol. 22, 015017 (2013). 